\title{
Velocity fields in breaking-limited waves on finite depth
}

\author{
John Grue, Jostein Kolaas and Atle Jensen \\ Mechanics Division, Department of Mathematics, University of Oslo, Norway \\ email: johng@math.uio.no \\ In memory of Enok Palm - teacher, colleague and friend
}

\begin{abstract}
The kinematics below the strongest possible periodic water waves on intermediate depth, for wave periods $T \sqrt{g / h}=8.75$ and 11.7 ( $g$ acceleration of gravity, $h$ water depth), is measured by PIV. The largest possible waves far away from the wave maker have a height of $H / h \simeq 0.49$ and a fluid velocity up to $0.5 \sqrt{g h}$ for these periods. Moderately breaking waves measured close to the wave maker have a turbulent surface region riding on top of a smooth flow with horizontal fluid velocity of $0.62 \sqrt{g h}$ at maximum, and wave height up to $H / h=0.63$. Strongly breaking waves have thicker turbulent surface region, smaller maximum height $(H / h=0.56)$ and horizontal fluid velocity of $0.72 \sqrt{g h}$ at maximum. Measurement of the flow below 72 breaking wave crests illustrate the range and variation of the elevation and kinematics. Experiments are compared to fully nonlinear and second order theories, where the former is valid for regular nonbreaking waves, and the latter gives conservative predictions for the very strong waves. Secondary streaming in the bottom boundary layer below the waves is measured.
\end{abstract}

Keywords:

\section{Introduction}

Development of wind farms in the offshore environment has made the subject of wave kinematics on finite and shallow water depth highly relevant. An example is the wind turbines on Sheringham Shoal north of Norfolk UK, where the water depth varies between 12 and $24 \mathrm{~m}$. A large wind farm on Dogger Bank in the southern part of the North Sea is currently being 
planned. The very large area of $17000 \mathrm{~km}^{2}$ has a depth between 15 and 36 $\mathrm{m}$. The longest waves that are of concern, also with the strongest energy density, have a period of about $T=12$ seconds according to information from industry. This means that the longest nondimensional wave period is in the range $T \sqrt{g / h} \sim 11-8$ on Sheringham Shoal and $T \sqrt{g / h} \sim 10-6$ on Dogger Bank, corresponding to an intermediate range of the water waves on finite depth ( $h$ water depth, $g$ acceleration of gravity). Several theories and analyses of waves on finite depth are available. The questions we address in the present experimental paper - not easily addressed by a theoretical approach - include:

1) How are the velocities in the strongest possible water waves on finite depth?

2) How do weak and strong breaking alter the velocities?

Measurements of the wave height $(H)$ of waves on finite, constant depth, in field observations, at coral reefs or other shallow locations at sea, as well as in laboratory experiments, show that this is $H / h=0.55$ at maximum [1]. The nonlinearity of the waves recorded in the field is commonly characterized by the parameter $F_{c}=(H / h)^{1 / 2}(T \sqrt{g / h})^{5 / 2}[2]$. The Ursell parameter may alternatively be used, see below. The maximum possible wave height is less than 0.55 in the regime where the waves are not characterized as shallow water waves, depending on the value of $F_{c}$. For example, the largest possible wave height is $H / h \simeq 0.49$ for $F_{c} \sim 150-300$, according to Nelson [1, figure 1]. This maximum height is relevant to the present experiments. Regular waves interacting with a reef of steep face and a weak, gradually decreasing reef-top slope, have a maximum value of $H / h$ that decreases when $F_{c}$ decreases [3]. Random wave heights that show initial Rayleigh distribution, transform during shoaling and breaking in the surf zone, resulting in wave height distributions that become nearly Rayleigh in the shallow water, a process that is characterized by some energy loss [4].

We note that a careful wave generation process may give larger wave heights than observed in the field and the vast majority of existing wave tank experiments. The important point is to postpone the inception of breaking. Zhang and Schäffer [5] used a stream function formulation to numerically generate periodic waves of heights $H / h=0.6$ for $F_{c}=340(T \sqrt{g / h}=11.4)$ and $H / h=0.55$ for $F_{c}=175(T \sqrt{g / h}=8.9)$. These elevations are 10$15 \%$ larger than the results summarized in $[1,2]$. Moreover, the results by 
Zhang and Schäffer are larger than what we have been able to generate in the present experiments for the similar wave periods. Yang et al. [6] employed second-order potential theory to calculate the far field waves with successful comparison to experiments, obtaining a similar maximum wave amplitude as Zhang and Schäffer [5]. A rapid, fully nonlinear-dispersive potential theory formulation in three dimensions, including wave generation, may be used to calculate nonbreaking waves on finite or variable water depth $[7,8]$.

The kinematics in steep periodic (regular) waves on finite water depth has been calculated recently using the method and code by Fenton [9]. Arguing that kinematics of the theoretically steepest waves of perfect symmetrical shape is not of practical relevance, calculations of the very large waves were avoided because of slow convergence properties of the method in this range [10]. This statement is correct, as the waves observed in the field do break and thus become limited in height. This saturation of the wave height is a key-point in the measurements we present in this paper and is further discussed below. Moreover, we perform numerical calculations using Fenton's method [9] with excellent correspondence to the (few) regular waves that are measured.

While the maximum wave height and propagation speed of waves on finite and shallow depth are extensively documented [1,11], see also further discussion in Section 1.1 below, there are gaps in the knowledge regarding the wave-induced kinematics. Attempts to obtain finite depth wave kinematics by hydrogen bubble method and hotfilm technique have been very little conclusive, particularly right below the water surface where also the fluid velocities are the strongest [12]. Waves that are strongly affected by breaking exhibit velocity fields that differ from waves that are regular. Weakly breaking waves, waves that may be characterized as extreme, or waves that are very high or break violently require very advanced computational strategies, see e.g. Iafrati [13]. The detailed effect of the breaking process and the resulting saturation invites for an experimental investigation of the wave-induced kinematics on finite water depth. The waves that are radiated away as well as the local wave breaking close to the wave maker are in the present experiments measured by Particle Image Velocimetry (PIV) with complementary elevation measurements by wave gauges. The present velocity field measurements complement previous measurements of wave kinematics on deep water $[14,15]$.

The paper is organized as follows. Following the Introduction, Section 2 describes the experimental set-up, wave motion and kinematics measure- 
ments far from the wave maker. Section 3 describes velocity fields in waves with moderate and strong breaking. Section 4 describes the jet induced by bottom boundary layer streaming. Summary and conclusions are given in the final Section 5 .

\subsection{Field observations of waves of maximum height, breaking on finite water depth and three-dimensional effects}

Before proceeding to the description of the kinematics experiments, a more detailed discussion of waves of maximum height and wave breaking on finite, constant water depth is given. In Nelson [1], a field measurement at John Brewer Reef was described, among others, obtaining wave measurements in four positions on the shallow horizontal reef at distances of $27 \mathrm{~m}$, $71 \mathrm{~m}, 120 \mathrm{~m}$ and $170 \mathrm{~m}$ from the reef edge. The water depth was in the range 1-3 $\mathrm{m}$, depending on the tide. The results in Nelson [1, figure 8] show that the ratio $H / h$ between the wave height and water depth could be up to 1 at the poles 1 and 2, and up to 0.8 at the pole number 3 . The observed wave height at the most inward measurement pole number 4 was up to $H / h \simeq 0.5$ for $F_{c} \simeq 160$. The observed wave height was somewhat less than 0.5 for the larger $F_{c} \simeq 300$. Nelson wrote in his paper that only two among the 72 measured events were marginally above his empirical curve of the maximum wave height, given by $(H / h)_{\max }=F_{c} /\left(22+1.82 F_{c}\right)$, which tends to $0.55 h$ when $F_{c}$ becomes large. For the intermediate wave range of moderate $F_{c}$, the maximum wave height was smaller than $0.55 h$.

A field measurement of breaking wind generated waves on finite, constant depth, undertaken by Babanin et al. [16], had a strongest wave record measured in $20 \mathrm{~m} / \mathrm{s}$ wind, with peak frequency of $0.36 \mathrm{~Hz}$ and a significant wave height of $H_{s}=0.41 h$. Besides investigating breaking properties and breaking thresholds, the up-crossing and down-crossing wave heights of the individual waves were recorded. Only 1.4 percent of these wave heights exceeded the $0.55 h$ height limit; all those waves were breaking, as documented by video recordings. In their conclusions, Babanin et al. [16] wrote that waves of height $0.44 h$ and higher always break, whereas waves with height less than $0.44 h$ may undergo random breaking and may either break or not.

The wave breaking observed in field experiments with and without the effect of wind may be caused by three-dimensional instabilities such as horseshoe instabilities, see McLean [17]. The class I and II instabilities of periodic waves on finite water depth were further calculated by Fructus et al. [18] finding that class II (horse shoe) instability dominates and leads to breaking of 
Stokes waves of wave slope $a k$ exceeding 0.13 , for $k h=1$ ( $a$ wave amplitude). This means that the horse shoe instability is present in wide wave fields when the wave height $2 a=H$ exceeds $0.26 h$ (for $k h=1$ ), relevant to the wave slope and water depth in the present experiments. Time simulations of wave growth beyond the instability threshold exhibit a local wave height of up to $0.45 h$ right before breaking, for $k h=1$ (Kristiansen et al. [19, figure 10a]). A stability analysis of class I and II instability of Stokes waves in shallow water with $k h<0.5$ was performed by Kharif and Francius [20]. Class I and II instabilities are not observed in the present narrow tank experiments, where also measurements are performed at a limited distance from the wave maker.

The apparent conflict between the limiting height of waves on finite constant depth and the maximum solitary wave amplitude of $0.83 h$ has been discussed in a paper by Massel [21]. His theoretical results for 2nd-, 3rd- and 4th-order approximations of cnoidal theory estimate a higher wave amplitude than Nelson's emprical formula. Massel also investigated the effect of a higher harmonic wave generation on the maximum height of the far field waves. In one example he obtained a second harmonic wave amplitude of up to 50 per cent of the first harmonic wave amplitude. In the present experiments we observe free higher harmonic wave components in the far field waves that are up to about 10 percent of the mother wave amplitude, however. Comparison between theoretical predictions of $(H / h)_{\max }$ to experimental observations of the same quantity in Massel [21, figure 6], shows 0.55 as the maximum for both theory and experiment. In that paper it was noted that the theoretical estimate of the experimental wave height could differ by more than 20 per cent.

In the present investigation the wave maker performs a sinusoidal oscillation (linear wave maker theory). The amplitude is gradually increased. We observe that the maximum wave height in the far field grows according to the amplitude of the wave maker, up to the level where breaking sets in. For stronger wave maker amplitude, the far field wave amplitude reduces, because of the breaking. With a wave generation where the wave breaking can be postponed, e.g. adding a second harmonic motion of the wave maker, the maximum far field wave amplitude can be somewhat enhanced, such as in Zhang and Schäffer [5] and Yang et al. [6]. They were able to numerically generate periodic waves of height $H / h=0.6$, similar to (about) the maximum wave height that Massel [21] indicated using estimates from potential theory, giving a far field amplitude that is 10-15 per cent higher than in field observations, e.g. Nelson [1] and Babanin et al. [16]. 
In the experiments detailed below we shall measure waves of maximum height in accordance with the observations in the field experiments reported by Nelson [1] and Babanin et al. [16]. The kinematics of the waves we measure is thus relevant to the waves observed in the field measurements. We calculate periodic waves of perfect symmetry using the method by Fenton [9]. Twenty terms are used giving good convergence. We have also computed waves of higher amplitude than what we are able to obtain in the experiments. An alternative accurate calculation procedure of the perfect symmetrical periodic waves is the method developed by Longuet-Higgins [23].

\section{Experiments}

\subsection{Laboratory set-up}

The experiments are performed in a $25 \mathrm{~m}$ long, $0.5 \mathrm{~m}$ wide wave tank in the Hydrodynamics Laboratory at University of Oslo. The water depth is 0.2 $\mathrm{m}$. Waves generated by a vertical piston at one end of the tank are recorded long before any reflection appears from the beach at the other end of the tank. Particle Image Velocimetry (PIV) is used to capture the wave-induced fluid velocities as well as the position of the wave surface. In order to get a sharp image of the surface elevation, the camera has a weak tilt upwards; Field Of View (FOV) of trapesoidal shape extending $0.206 \mathrm{~m}$ in the vertical has upper side $0.21 \mathrm{~m}$ long and lower side $0.198 \mathrm{~m}$ long. The resolution is 1024 by 1024 pixels.

The number of frames per second is 1500 for nonbreaking waves and 200 for breaking waves; 6000 frames are recorded in each series. The time interval between the images is determined by an optimal accuracy of the PIV-analysis.

PIV recordings are taken at positions $6.93 \mathrm{~m}, 4.43 \mathrm{~m}$ and $2.08 \mathrm{~m}$ from the wave maker. Ultra sound probes are used for additional measurement of the surface excursion.

\subsection{Waves far from the wave maker}

Periodic waves of largest possible elevation propagating along a fluid layer on a horizontal bottom are studied. The waves are characterized by the wave period $T$ and wave height $H$, where the latter is measured by the troughto-crest height. The water depth $h$ is measured when there are no waves. Two slightly different wave periods, $T \sqrt{g / h}=8.75$ and 11.7 , in a range corresponding to the longest waves that are relevant for offshore wind energy 
applications, are considered. Waves of these periods have maximum height far away from the generation site of $H / h \simeq 0.49$, see Nelson [1, figure 1] and is also obtained in the present experiments.

Experiments where the wave paddle amplitude is gradually increased from small to large excursion, beyond the level where wave breaking starts, have been studied. Wave generation that covers the range from weak to strong breaking is reported here. Incipient wave breaking occurs about one wave length away from the wave maker. The breaking point moves towards the wave maker according to the increased strength of the wave generation. The waves radiating out reach a saturation of the wave height corresponding to a maximum value. Still stronger wave maker amplitude reduces the elevation of the far field wave train, because of the stronger breaking close to the wave maker. The wave breaking process and the finite excursion of the wave maker generate additional short higher harmonic waves riding on top of the dominant motion. The short waves arrive at the recording position later than the main wave (figure 1). We note that the velocity color plots in figures 1-2 are composed by measurements from several repeated experiments, causing small discontinuities (and white lines) to appear in the plots.

\subsection{Regular wave}

The elevation history of the wave train for wave period $T \sqrt{g / h}=8.75$ recorded at position $4.43 \mathrm{~m}$ from the wave maker is illustrated in figure 1a. The wave maker amplitude is $\xi_{0} / h=0.267$. The first three wave crests of the wave train occur at 4.65, 5.9 and 7.15 seconds, respectively. The crests are observed before the higher harmonic waves appear. The third relatively symmetrical crest has a trough-to-crest height of $H / h=0.44$ and crest-totrough height of 0.43 . The average wave height of 0.435 is 13 per cent smaller than the maximum possible wave height of 0.49 for this wave period.

The horizontal particle velocity below the third wave crest is up to $u / \sqrt{g h}=$ 0.363 (below crest) (figure 2a) $(6.4 \mathrm{sec} .<t<7.6 \mathrm{sec}$.). The horizontal velocity at the surface level, at the troughs before and after the crest, is down to $u / \sqrt{g h}=-0.171$ and -0.174 , respectively. Calculations using Fenton's method [9] for periodic waves with $T \sqrt{g / h}=8.75$ and $H / h=0.435$ give $u / \sqrt{g h}=0.365$ below crest and $u / \sqrt{g h}=-0.135$ at trough. Except for the latter theoretical value, which is somewhat less negative than in measurement, the theoretical computations fit very well to the experimental, regular wave (figure $2 \mathrm{~b}$ ). A number of 20 terms are retained in the calculations using Fenton's method. 
A notable difference between the experimental (figure 2a) and theoretical wave (figure $2 \mathrm{~b}$ ) is the increased velocity close to the sea bottom, for $-0.996<y / h<-0.95$, where a forward moving jet is superposed on the wave-induced velocity, see further discussion in Section 4 below.

\subsection{Waves of maximum height}

The nonlinearity of the wave making, including the weak or strong breaking taking place about one wavelength away from the wave maker, as well as the finite excursion of the wave maker, generate free higher harmonic waves riding on top of the dominant wave motion. The higher harmonic amplitudes become saturated if the nonlinearity is strong enough, Grue [22].

The higher harmonic waves add to the total wave height and become present in the elevation measurements from the fourth wave crest at 8.5 seconds and onwards, at the recording position at $4.43 \mathrm{~m}$, for the wave of period $T \sqrt{g / h}=8.75$ (figure 1a). For the longer wave, with $T \sqrt{g / h}=$ 11.7, recorded at $6.93 \mathrm{~m}$, the second harmonic waves propagate at about the same speed as the main wave, arriving about simultaneously at the recording position (figure $1 b$ ). The resulting maximum wave height of $H / h=0.49$ for $T \sqrt{g / h}=8.75$ and $H / h=0.48$ for $T \sqrt{g / h}=11.7$ correspond very well to previous laboratory measurements and field observations.

The nonlinearity parameter of these waves are $F_{c}=(H / h)^{1 / 2}(T \sqrt{g / h})^{5 / 2}=$ 159 for $T \sqrt{g / h}=8.75$ and $H / h=0.49$, and $F_{c}=324$ for $T \sqrt{g / h}=$ 11.7 and $H / h=0.48$, see Nelson [1, figure 1]. Alternatively, the ratio between nonlinearity and dispersion is evaluated by the Ursell parameter, $U r=(H / h) /(k h)^{2}=0.795$ and $U r=1.50$ for the two waves, respectively.

\subsubsection{Wave with $T \sqrt{g / h}=8.75$}

The trough-to-crest heights of the eight wave crests of period $T \sqrt{g / h}=$ 8.75, from 8 to 17.5 seconds in figure 1a, have a maximum of $H / h=0.49$, average value of $\bar{H} / h=0.47$ and a standard deviation of 3 per cent. The measured horizontal velocity below wave crests 4 to 11 is $u / \sqrt{g h}=0.499$ at maximum (figure 2c). This is 33 per cent higher than the velocity below wave crest number three (figure 2a). The horizontal velocity below wave crests 4 to 11 in figure 1a have an average value of $u / \sqrt{g h}=0.481$ and standard deviation of $4.7 \%$. The horizontal fluid velocities at the troughs (at the surface level) preceeding crests 4 to 11 have an average value of $u / \sqrt{g h}=-0.140$ (standard deviation of 4.6 per cent) and is comparable to the theoretical value of -0.135 in the periodic theoretical wave, but is slightly 
weaker than the nondimensional velocity of -0.171 at the trough preceeding the third wave crest.

Horizontal velocity profiles at 40 subsequent time steps with $\Delta t \sqrt{g / h}=$ 0.1821 further illustrate the stronger kinematics below the crest of wave 10 (figure 3a). Computation of the similar velocity profiles using Fenton's method with $H / h=0.49$ and $T \sqrt{g / h}=8.75$ as input illustrate that the theoretical wave has velocity $u / \sqrt{g h}$ up to 0.42 at crest which is $16 \%$ smaller than in the experimental wave. The higher harmonic waves that are present in the experimental waves are obviously responsible for the increased velocity at crest, as well as the increase in wave height, taking place from wave crest number 4 in this measurement series (see figure 1a). The effect of the higher harmonic waves on the $u$-velocity is also apparent close to the surface, see figure $3 \mathrm{a}$, plot i.

The velocity field below $y / h=-0.4$ experiences only very minor change due to the higher harmonic waves. The fluid velocity well above the boundary layer at the bottom oscillates between $-0.13<u / \sqrt{g h}<0.16$. Regarding the vertical velocity, the higher harmonic waves exhibit very small effect and are hardly observed in the results (figure 4).

Waves 4 to 11 ( 8 to 17.5 seconds) show, very close to the bottom $(-0.996<$ $y / h<-0.95)$ a forward moving jet superposed on the wave-induced velocity field (figure 3a, plots iii-iv). This motion, also present below wave crest 3, has now reached a steady state, see further discussion in Section 4 below.

\subsubsection{Wave with $T \sqrt{g / h}=11.7$}

The wave train with $T \sqrt{g / h}=11.7$ in figure $1 \mathrm{~b}$ shows a maximum height of $H / h=0.48$, average height of $\bar{H} / h=0.47$ and a standard deviation of 1.6 per cent, for the crests recorded from 8 to 25 seconds. The measured kinematics below wave crest number 11 is representative for these waves and shows that the horizontal velocity slightly exceeds $u / \sqrt{g h}=0.4$ at maximum (figures 2c, 3c). Computation of this wave with $H / h=0.48$ shows about similar velocities. Note that the computational crest and trough appear at higher values $\left(y_{\max } / h=0.37, y_{\min } / h=-0.12\right)$ than in experiment $\left(y_{\max } / h=\right.$ $\left.0.31, y_{\min } / h=-0.17\right)$. The difference in level above the mean water line enhances the kinematics in the computation, in the top part of the wave. We have therefore made another computation where the reference depth $\left(h^{*}\right)$ is increased by $5 \%$ and height and period accordingly reduced to $H / h^{*}=$ 0.466 and $T \sqrt{g / h^{*}}=11.4$, respectively, giving $u \sqrt{g h^{*}}=0.37$ (maximum), $y_{\max } / h^{*}=0.35$ and $y_{\min } / h^{*}=-0.12$. Although we have sought the position 
along the wave tank where the trough-to-crest height is the largest for this train, the comparison between experiment and calculation illustrates that the higher harmonic waves reduce the crests and increase the troughs, in the experimental wave train with period $T \sqrt{g / h}=11.7$.

\subsection{Comparison to second order theory}

Second order wave theory is extensively used in engineering analysis of offshore structures on finite and infinite water depth. Second order irregular wave theory is relatively easy to implement, easy to use in practice and rapid. The relevance of second order irregular wave theory in obtaining the elevation and kinematics of strong waves on finite water depth may be of interest.

The first and second order velocity potentials of Stokes waves on finite water depth give the following horizontal velocities

$$
u_{I}^{(1)}=\frac{g k A}{\omega} \frac{\cosh k(y+h)}{\cosh k h} \cos \chi, \quad u_{I}^{(2)}=\frac{3 \omega k A^{2} \cosh 2 k(y+h)}{4 \sinh ^{4} k h} \cos 2 \chi,
$$

where $\chi=k x-\omega t+$ phase, $\omega^{2}=g k \tanh k h$ and $(x, y)$ are horizontal and vertical coordinates with $y=0$ at the water level at rest.

For the conditions in the present experiments with height $2 A / h=0.49$ and period $T \sqrt{g / h}=8.75$ we obtain $u_{I}^{(1)}+u_{I}^{(2)}=0.52 \sqrt{g h}$ at crest; the similar value is $-0.16 \sqrt{g h}$ at trough, see figure $3 \mathrm{f}$.

For the wave with $T \sqrt{g / h}=11.7$ (and $2 A / h=0.49$ ) we obtain $u_{I}^{(1)}+$ $u_{I}^{(2)}=0.54 \sqrt{g h}$ at crest and $-0.09 \sqrt{g h}$ at trough.

It is evident that the second order theory is only moderately conservative for the surface velocities of the shorter experimental wave (figure 3a). It becomes quite conservative for the longer wave as well as near the bottom (figure $3 \mathrm{a}, \mathrm{c}$ ).

We note that irregular waves observed in field experiments, such as the wind waves in the investigation by Babanin et al. [16], have a maximum wave height of $0.55 h$ and that waves of height exceeding $0.44 h$ all break, see Section 1.1 of the paper. A possible method to estimate the kinematics in irregular waves is to let the amplitude $A$ in (1) be non-constant, where the second order contribution and the wave induced velocity field can be obtained as in Trulsen et al. [23, eqs. (49)-(52)]. An example of the excellent performance of this theory can be found in Trulsen et al. [24, figure 1]. 


\section{Motion close to the wave maker}

In this section we discuss three series of wave measurements, each including 24 wave crests. The waves are recorded close to the wave maker at position $2.08 \mathrm{~m}$, for period $T \sqrt{g / h}=8.75$. Wave maker amplitudes of $\xi_{0} / h=0.288,0.329$ and 0.370 are stronger than for the waves discussed in the previous sections, with $\xi_{0} / h=0.267$. The wave motion exhibits moderate and strong breaking and is visible in the form of a band corresponding to a turbulent region between the wave surface and a region below where the flow is smooth. The band with breaking turbulent flow is indicated by light color and the region with smooth flow by dark color, see figure 5 . The wave induced velocities are obtained in the region with smooth flow. We have not been able to obtain velocities in the turbulent region. The boundary between the laminar (smooth flow) and turbulent regions in figure 5 is determined by where it has been possible to obtain convergent velocity vectors.

\subsection{Waves with moderate breaking}

Consider the weakest wave series in figure $5 \mathrm{c}\left(\xi_{0} / h=0.288\right)$. The troughto-crest height has a maximum of $H / h=0.63$, average height of $\bar{H} / h=0.58$ and a standard deviation of $4.7 \%$. The elevation and velocities recorded through one of the wave periods of this series (4.7 to 5.9 seconds) exhibit a relatively symmetrical appearance, see figure 6a,b. The elevation (black line) and velocities (color plots) are both obtained from the images of the flow used for the subsequent PIV-analysis where the white gap indicates the region where wave breaking and turbulent motion are taking place.

The horizontal velocities (figure 6a) are quite similar to the largest far field wave shown in figure $2 \mathrm{c}$, with the important exception, that the $u$-velocity right below the breaking region, right below the wave crest, is $u / \sqrt{g h}=0.6$ at maximum. This is 20 per cent higher than in the wave in figure $2 \mathrm{c}$. The vertical velocities (figure 6b) are somewhat smaller than in the largest far field wave (figure 4b).

The horizontal velocity profiles below the 24 crests in the series show that $u / \sqrt{g h}=0.62$ at maximum, see figure $6 \mathrm{c}$. The standard deviation, indicated in the figure, shows that the maximum velocity of $u / \sqrt{g h} \simeq 0.62$ occurs in only a few among the 24 measured velocity profiles. The majority of the velocity profiles exhibit $u / \sqrt{g h} \simeq 0.5 \pm 0.05$ in the top of the smooth flow, right below crest. For $y / h<0.2$, the velocity profile (at maximum) is quite

similar to the velocity profile of the far field wave (at maximum) 
Besides the turbulent region right below the surface, the breaking introduces perturbations and sends air bubbles into the flow in the smooth region. The higher harmonic oscillations are illustrated in the velocity profiles at time intervals $\Delta t \sqrt{g / h}=1.75$ (figure 8a).

\subsection{Waves with strong breaking}

The wave motion for wave making amplitude of $\xi_{0} / h=0.370$ exhibits a reduced height, with $H / h=0.56$ at maximum. Averaging over all crests, the mean height is 0.51 with a standard deviation of $3.7 \%$. Elevation and $u$-velocities for time frames $11 \mathrm{~s}<t<12.2 \mathrm{~s}$ (figure 7a) and $8.5 \mathrm{~s}<t<9.6$ $\mathrm{s}$ (figure $7 \mathrm{~b}$ ) show that the velocites below crest are larger and wave profile more asymmetrical. Further, the white band between the elevation (black line) and the smooth region is thicker for the strongly breaking waves. The white band indicating the turbulent region of the flow appears asymmetrically along the wave, where its main extension is behind the wave crest. Attempts to estimate the velocity of the front and back faces of the turbulent region indicate that these are smaller than the wave velocity, although measurements are inaccurate.

The velocity profiles below the 24 crests show that $u / \sqrt{g h}=0.72$ at maximum (figure $7 \mathrm{c}$ ). The figure shows that $u \sqrt{g h}$ increases from 0.25 at the average water level $(y=0)$ to 0.72 at $y / h=0.18$ indicating a very strong velocity gradient in this flow. It is evident that the very strong increase in the velocity above the mean water level is compensated by a corresponding reduction in the velocity below $y=0$. The ensemble of the 24 velocity profiles indicates that $u / \sqrt{g h}=0.6 \pm 0.05$ is a typical picture of the velocity maxima of several crests (figure $7 \mathrm{c}$ ).

For the somewhat weaker excitation with wave paddle amplitude of $\xi_{0} / h=$ 0.329 the horizontal velocity in the corresponding ensemble of 24 crests is up to $u / \sqrt{g h}=0.65$ (figure $7 \mathrm{~d}$ ). This somewhat weaker generation and breaking wave motion exhibit less strong velocity gradient in the upper part of the velocity profile compared to the stronger case in figure $7 \mathrm{c}$. The velocities below the mean water level are less reduced, moreover. The ensemble of the 24 velocity profiles indicates that $u / \sqrt{g h}=0.35 \pm 0.07$ is a typical picture of the velocity maxima of several crests (figure $7 \mathrm{~d}$ ).

The breaking wave motion of the strong wave shown in figure $7 \mathrm{a}$, for $11 \mathrm{~s}<t<12.2 \mathrm{~s}$, introduces strong perturbations to the smooth flow, as visualised by the velocity profiles in the front and back face, in the upper part of the wave, for consecutive times with $\Delta t \sqrt{g / h}=1.75$, see figure 8c. 
The stronger breaking wave (figure 7b) measured for time $8.5 \mathrm{~s}<t<9.6 \mathrm{~s}$ shows even stronger wiggles, particularly in the back face of the wave. The velocity profiles show very strong vertical oscillations both in time and along the vertical, see figure 8c.

\section{Bottom boundary layer streaming}

The recordings of the wave train at $4.43 \mathrm{~m}$ (and $6.93 \mathrm{~m}$ ) exhibit a forward moving jet very close to the bottom. This jet is superposed on the waveinduced velocity field and is driven in the form of a streaming outside the oscillatory bottom boundary layer. The jet appers in the wave train shown in figure 1a from wave 3 and onwards and occurs for $-0.996<y / h<-0.95$. The $u$-velocity at $y / h=-0.95$ becomes, after an initial transiental behaviour, $u / \sqrt{g h} \simeq 0.015+0.145 \cos (\omega t+$ phase $)$, while at $y / h=-0.996$ the velocity is higher, with an incremental velocity of $\Delta u / \sqrt{g h} \simeq 0.028+$ $0.028 \cos (\omega t+$ phase) (figure 9$)$. Measurement of the latter has been obtained in close-up recordings with FOVs of $25 \mathrm{~mm}$ by $25 \mathrm{~mm}$ width and height, at the tank bottom, corresponding to a vertical (and horizontal range) of $-1<y / h<-0.9875$. The average value of the incremental velocity is explained by the streaming induced by the oscillatory boundary layer at the fluid bottom. The boundary layer thickness in this experiment is

$\sqrt{2 \omega / \nu} / h \simeq 0.002(T \sqrt{g / h}=8.75)$. According to Longuet-Higgins [23] the streaming velocity outside the boundary layer is $\bar{u}=(5 / 4) U_{0}^{2} / c$ where $U_{0}$ is the velocity amplitude of the oscillatory wave-induced fluid velocity outside the boundary layer and $c=\omega / k$ denotes wave speed. With $U_{0} / c_{0}=0.145$ (from figure $3 \mathrm{a}$ ) we obtain $\bar{u}=(5 / 4) U_{0}^{2} / c \simeq 0.028$ which agrees with the measurements.

\section{Summary and conclusions}

In this paper we have addressed two questions: 1) How are the velocities in the strongest possible water waves on finite depth? 2) How do weak and strong breaking alter the velocities?

We have assumed for the waves we study that the bottom is horizontal. The story may be different for a tilted bottom.

An important dimension is that wave breaking limits the wave height, which in nondimensional terms is up to 0.55 , according to observations in the field and in wave tank experiments. In the intermediate depth range that we 
have investigated here, for two nondimensional wave periods, $T \sqrt{g / h}=8.75$ and 11.7 ( $T$ period, $g$ acceleration of gravity, $h$ water depth), the maximum wave height is $H / h \simeq 0.49$ and is in accordance with results from several field and laboratory measurements summarized by Nelson [1, figure 1]. The nondimensional periods in the present experiments were chosen because of the relevance to offshore wind turbine applications in finite water depth.

The periodic wave train far away from the wave maker reaches a steady state where small higher harmonic free waves - generated by combination of breaking or local steepening about one wave length away from the wave maker, as well as by the finite excursion of the wave maker - ride on top of the dominant wave motion. It is assumed that this scenario is representative for the real waves on the ocean. As for the main wave, the higher harmonic waves become saturated in amplitude [22]. It is evident that the higher harmonic waves alter the crest and trough levels as well as the kinematics of the wave field. For the wave train with $T \sqrt{g / h}=8.75$ and $H / h=0.49$ we find that the horizontal fluid is $u / \sqrt{g h}=0.5$ at maximum. This is 16 per cent higher than fully nonlinear calculations by Fenton's method [9] for the same wave, assuming periodic waves without any parasittic effects - we have used 20 terms in Fenton's method in these calculations. In the leading part of this wave train, before the higher harmonic waves arrive at the measurement position, we have an almost perfect match between the experimental wave and the calculations $(T \sqrt{g / h}=8.75, H / h=0.435)$.

For the other wave train $(T \sqrt{g / h}=11.7, H / h=0.48)$ the horizontal velocity is up to $u / \sqrt{g h}=0.4$ in the experiments. Nonlinear calculations give about the same kinematics; the nominal crest and trough values are somewhat larger in the theoretical wave compared to experiment. Results for the experimental velocity profiles throughout the wave periods are given.

It is noted that waves of greater height than found here - our maximum waves correspond to findings in several data sets [1] - may be obtained by a special, careful wave generation, giving $H / h=0.55$ for $T \sqrt{g / h}=8.9$ and $H / h=0.6$ for $T \sqrt{g / h}=11.4$ [5]. We also note, that by careful wave generation, the higher harmonic waves in very strong waves may be removed [6].

Moderately and strongly breaking waves were investigated at a position corresponding to 10 water depths away from the wave maker. Waves with moderate breaking attain a maximum wave height of $H / h=0.63$. Elevation and fluid velocities are relatively symmetrical about the wave crests. A 
turbulent region rides on top of the smooth flow where the horizontal fluid velocity is $u / \sqrt{g h}=0.62$ at maximum. The majority of the velocity profiles below 24 wave crests exhibit $u / \sqrt{g h} \simeq 0.5 \pm 0.05$ in the top part of the smooth flow, right below crest. The wave breaking generates higher harmonic oscillations of the flow in the smooth region and sends air bubbles into this region.

Waves with strong breaking show a maximum wave height of 0.56 . The elevation is forward leaning and the fluid velocities as well as the turbulent region of the flow appears asymmetrically about the crest. An ensemble of 24 velocity profiles indicates that $u / \sqrt{g h}=0.6 \pm 0.05$ is typical of the velocity maxima of several crests, and that the horizontal velocity maximum is $u / \sqrt{g h}=0.72$ in one of the waves. Another ensemble of 24 velocity profiles, with somewhat weaker generation, still with strong breaking, indicates that $u / \sqrt{g h}=0.35 \pm 0.07$ is typical of the velocity maxima of several crests, and that the horizontal velocity maximum is $u / \sqrt{g h}=0.65$ in one of the waves. The waves with strong breaking exhibit very strong velocity gradient in the upper part of the smooth flow, below the turbulent region. The very strong increase in the velocity above mean water level is compensated by a reduction in the velocity below this level. The strong breaking introduces strong perturbations to the fluid velocities, particularly in the back face of the wave.

Regarding computation of the experimental waves there are several options. While the breaking waves may be difficult to compute, the nonbreaking waves far away from the generation site - in the present experiments with a periodic generation - are best computed by fully nonlinear theory, where Fenton's [9] program is a clear option. Input to the periodic wave calculations should be the maximum wave height according to Nelson [1, figure 1]. The periodic calculations do not include the effect of the small higher harmonic waves that typically are present in the maximum experimental waves. We have not tested out Stokes fifth order theory. Second order theory may be relevant for engineering applications, particularly for irregular wave calculations. We find here that second order Stokes wave theory gives conservative estimates of the fluid velocities in the present periodic very large waves.

The present PIV measurements show velocities in the laminar boundary layer - driven by the periodic wave-induced bottom velocity - corresponding to a streaming outside the boundary layer, in the form of a forward pointing jet, with an average velocity corresponding to laminar boundary layer theory. 
Acknowledegement This research was funded by the Research Council of Norway through NFR 191204/V30, Wave-current-body-interaction.

\section{References}

[1] R. C. Nelson, Depth limited design wave heights in very flat regions. Coastal Engineering 23 (1994) 43-59.

[2] D. H. Swart and C. C. Loubser, Vocoid theory for all nonbreaking waves. Proc. 16th Coastal Eng. Conf. 1 (1978) 467-486.

[3] M. R. Gourlay, Wave transformation on a coral reef, Coastal Engineering 23 (1994) 17-42.

[4] E. B. Thornton and R. T. Guza, Transformation of wave heigth distribution, J. Geophys. Res. 88(C10) (1983) 5922-38.

[5] H. Zhang and H. Schäffer, Approximate stream function wavemaker theory for highly nonlinear waves in wave flumes. Ocean Engng. 34 (2007) 1290-1302.

[6] Z. Yang, S. Liu, H.B. Bingham and J. Li, Second-order theory for coupling 2D numerical and physical wave tanks: Derivation, evaluation and experimental validation. Coastal Engineering 71 (2013) 37-51.

[7] D. Clamond, D. Fructus, J. Grue and Ø. Kristiansen, An efficient model for three-dimensional surface wave simulations. Part II Generation and absorption. J. Comput. Phys. 205 (2005) 686-705.

[8] D. Fructus and J. Grue, An explicit method for the nonlinear interaction between water waves and variable and moving bottom topography. J. Comput. Phys. 222 (2007) 720-739.

[9] J. D. Fenton, The numerical solution of steady water wave problems. Computers \& Geosciences 14(3) (1988) 357-368.

[10] D. Clamond, Note on the velocity and related fields of steady irrotational two-dimensional surface gravity waves. Phil. Trans. R. Soc. A 370 (2012) 1472-1586. 
[11] M. Tissier, P. Bonneton, R. Almar, B. Castelle, N. Bonneton and A. Nahon, Field measurements and non-linear prediction of wave celerity in the surf zone. Eur. J. Mech. B/Fluids 30 (2011) 635-641.

[12] Y. Iwagaki and T. Sakai, Horizontal water particle velocity of finite amplitude waves. Proc. 12th Conf. Coastal Engng. 1 (1970) 309-325.

[13] A. Iafrati, Numerical study of the effects of the breaking intensity on wave breaking flows. J. Fluid Mech. 622 (2009) 371-411.

[14] J. Grue, D. Clamond, M. Huseby and A. Jensen, Kinematics of extreme waves in deep water. Appl. Ocean Res. 25 (2003) 355-366.

[15] J. Grue and A. Jensen, Orbital velocity and breaking in steep random gravity waves. J. Geophys. Res. 117 (2012) C07013.

[16] A.V. Babanin, I.R. Young, M.L. Banner, Breaking probabilities for dominant surface waves on water of finite constant depth. J. Geophys. Res. 106(C6) (2001) 11659-11676.

[17] J.W. McLean, Instabilities of finite-amplitude gravity waves on water of finite depth. J. Fluid Mech. 114 (1982) 331-341.

[18] D. Fructus, C. Kharif, M. Francius, Ø. Kristiansen, D. Clamond and J. Grue, Dynamics of crescent water wave patterns. J. Fluid Mech. 537 (2005) 155-186.

[19] Ø. Kristiansen, D. Fructus, D. Clamond and J. Grue, Simulations of crescent water wave patterns on finite depth. Phys. Fluids. 17 (2005) 064101.

[20] C. Kharif and M. Francius, Three dimensional instabilities of periodic gravity waves in shallow water. J. Fluid Mech. 561 (2006) 417-437.

[21] S.R. Massel, On the largest wave height in water of constant depth. Ocean Engng. 23(7) (1996) 553-573.

[22] M.S. Longuet-Higgins, Lagrangian moments and mass transport in Stokes waves Part 2. Water of finite depth. J. Fluid Mech. 186 (1988) 321-336. 
[23] J. Grue, Nonlinear water waves at a submerged obstacle or bottom topography. J. Fluid Mech. 244 (1992) 455-476.

[24] K. Trulsen, O.T. Gudmestad and M.G. Velarde, The nonlinear Schrödinger method for water wave kinematics on finite depth. Wave motion 33 (2001) 379-395.

[25] K. Trulsen, A. Jensen and J. Grue, Water wave kinematics of steep irregular waves - systematic perturbation approach, empirical law, PIV measurements and engineering practice. In: Proc. 20th Int. Workshop on Water Waves and Floating Bodies, edited by J. Grue, Longyearbyen, 29 May-1 June, 2005.

[26] M. S. Longuet-Higgins, Mass transport in water waves. Phil. Trans. Roy. Soc. Lond. Ser. A, Mathematical and Physical Sci. 245(903) (1953) 535581.

Figure Captions

Figure 1.

Elevation $\eta / h$ and horizontal velocity $u / \sqrt{g h}$ (color scale) by PIV. a) at $4.43 \mathrm{~m}$ for $T \sqrt{g / h}=8.75$, wave maker amplitude $\xi_{0} / h=0.267$ and $2 \mathrm{~s}<t<$ $17.7 \mathrm{~s} . \mathrm{b})$ at $6.93 \mathrm{~m}$ for $T \sqrt{g / h}=11.7$, wave maker amplitude $\xi_{0} / h=0.360$ and $4.2 \mathrm{~s}<t<25 \mathrm{~s}$.

Figure 2 .

Elevation $\eta / h$ and horizontal velocity $u / \sqrt{g h}$ (isolines/color scale) by PIV. a) Same as Figure 1a, for $6.5 \mathrm{~s}<t<7.75 \mathrm{~s}, \mathrm{~b})$ Computation (Fenton's method) of wave in a), c) Same as Figure 1a, for $15.25 \mathrm{~s}<t<16.5 \mathrm{~s}$, d) Same as Figure 1b, for $20.9 \mathrm{~s}<t<22.3 \mathrm{~s}$.

Figure 3 .

Velocity profiles $u / \sqrt{g h}$ vs. $y / h$. a) Experimental wave in Figure 2c), $\Delta t \sqrt{g / h}=0.1821$ (upper front (i), upper back (ii), lower front (iii), lower back (iv)). b) Computation wave in a). c) Experimental wave in Figure 2d), $\Delta t \sqrt{g / h}=0.2430$ (upper front (i), upper back (ii), lower front (iii), lower back (iv)). d) Computation of wave in c), with $H / h=0.48, T \sqrt{g / h}=11.7$. e) Computation of wave in c), with $H / h^{*}=0.466, T \sqrt{g / h^{*}}=11.4$. f) Velocity profiles at crest and trough by second order theory, $2 A / h=0.49$. $T \sqrt{g / h}=8.75$ (thick dots), $T \sqrt{g / h}=11.7$ (line). 
Figure 4 .

Vertical velocity $v / \sqrt{g h}$ (isolines/color scale) by PIV at $4.43 \mathrm{~m} . T \sqrt{g / h}=$ 8.75. a) $6.5 \mathrm{~s}<t<7.75 \mathrm{~s}$, b) $15.25 \mathrm{~s}<t<16.5 \mathrm{~s}$.

Figure 5 .

PIV recordings of breaking waves at $2.08 \mathrm{~m} . T \sqrt{g / h}=8.75 .24$ crests in each series. Smooth flow (dark). Breaking turbulent flow (light). Wave maker amplitude: a) $\xi_{0} / h=0.3697$, b) $\xi_{0} / h=0.3287$, c) $\xi_{0} / h=0.2876$.

Figure 6 .

Elevation and $u / \sqrt{g h}$ (a) and $v / \sqrt{g h}$ (b) (color scale) for crest number three in figure $5 \mathrm{a}(4.7 \mathrm{~s}<t<5.95 \mathrm{~s})$. Moderately breaking wave. $\mathrm{c})$ Ensemble average (thick line) and standard deviation (thin lines) of all velocity profiles $u / \sqrt{g h}$ vs. $y / h$ below the 24 crests in figure $5 \mathrm{c}$.

Figure 7.

Same as figure 6a but for strongly breaking wave series. a) crest 8 figure $5 \mathrm{a}(11 \mathrm{~s}<t<12.25 \mathrm{~s})$, b) crest 6 in figure $5 \mathrm{a}(8.6 \mathrm{~s}<t<9.75 \mathrm{~s})$. c) same as figure $6 \mathrm{c}$ but for series in Figure 5a. d) same as figure $6 \mathrm{c}$ but for series in Figure $5 \mathrm{~b}$.

Figure 8.

Horizontal velocity profiles at time intervals $\Delta t \sqrt{g / h}=1.75$. (i) front, (ii) back. Wave in a) Figure 6a. b) Figure 7a. c) Figure 7b.

Figure 9 .

$u / \sqrt{g h}$ at $y / h=-0.96(--)$ and $y / h=-0.996(-)$ vs. time at the bottom, below the waves in figure 1a. 
a)

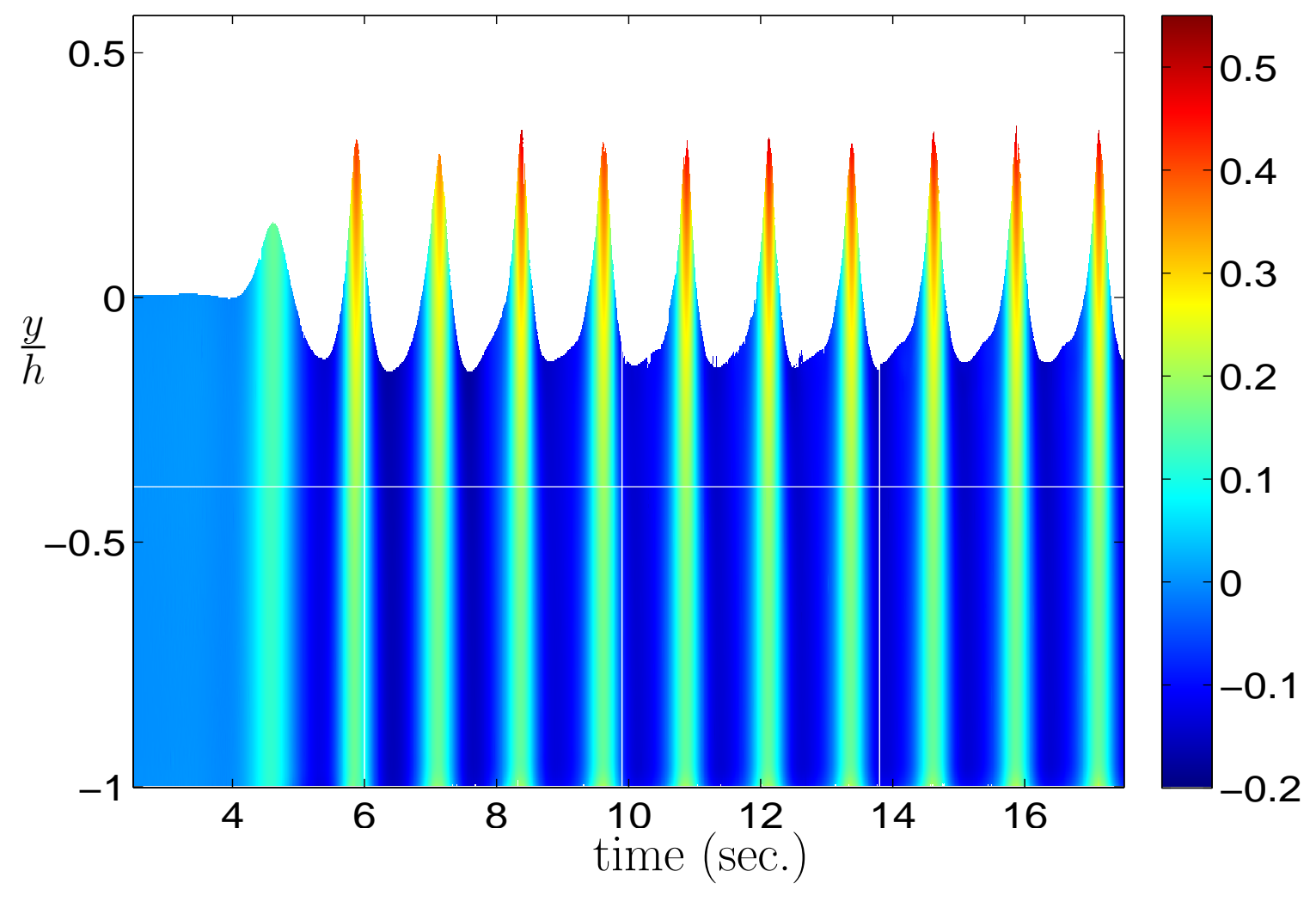


b)

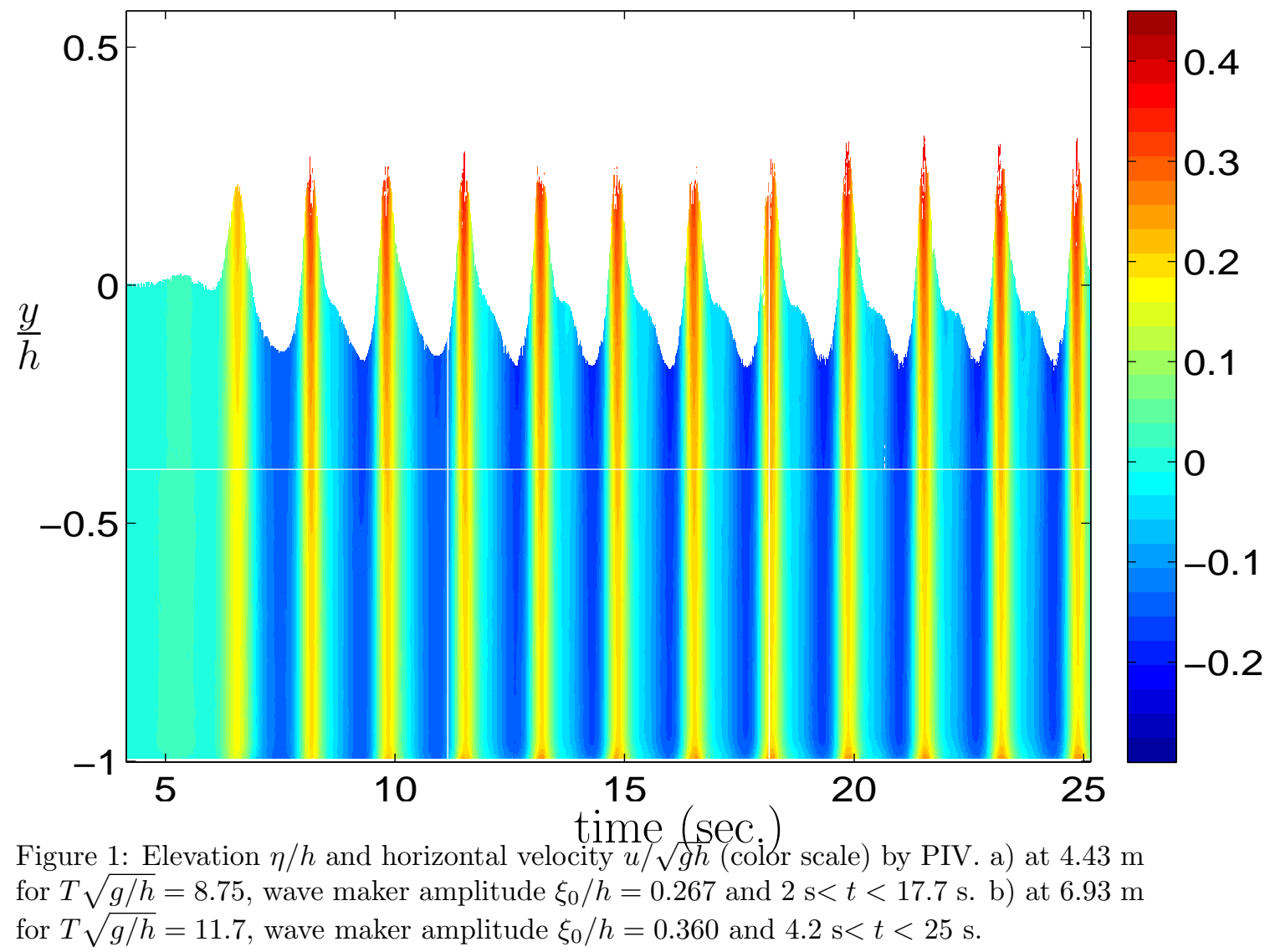


a)
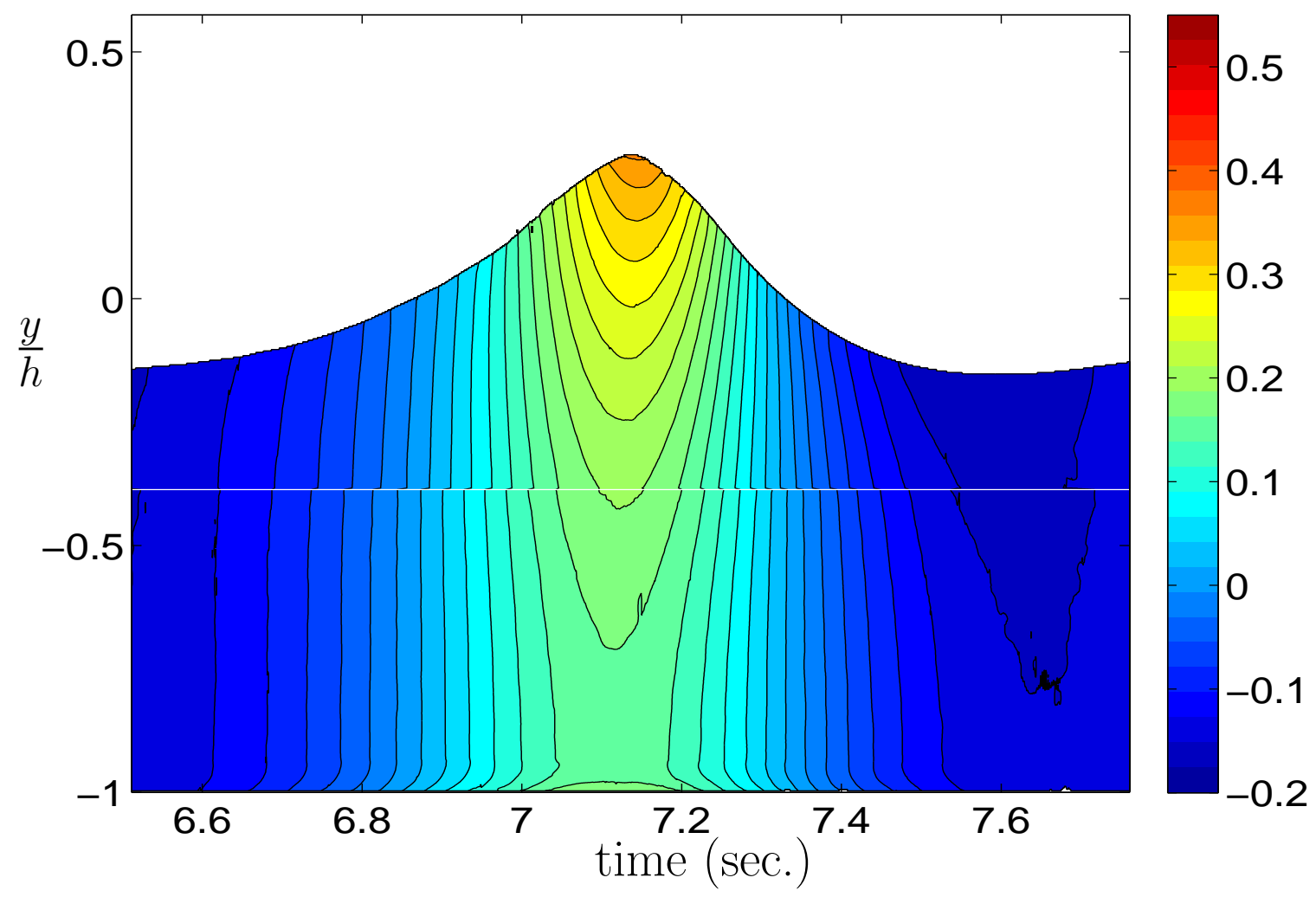

b)

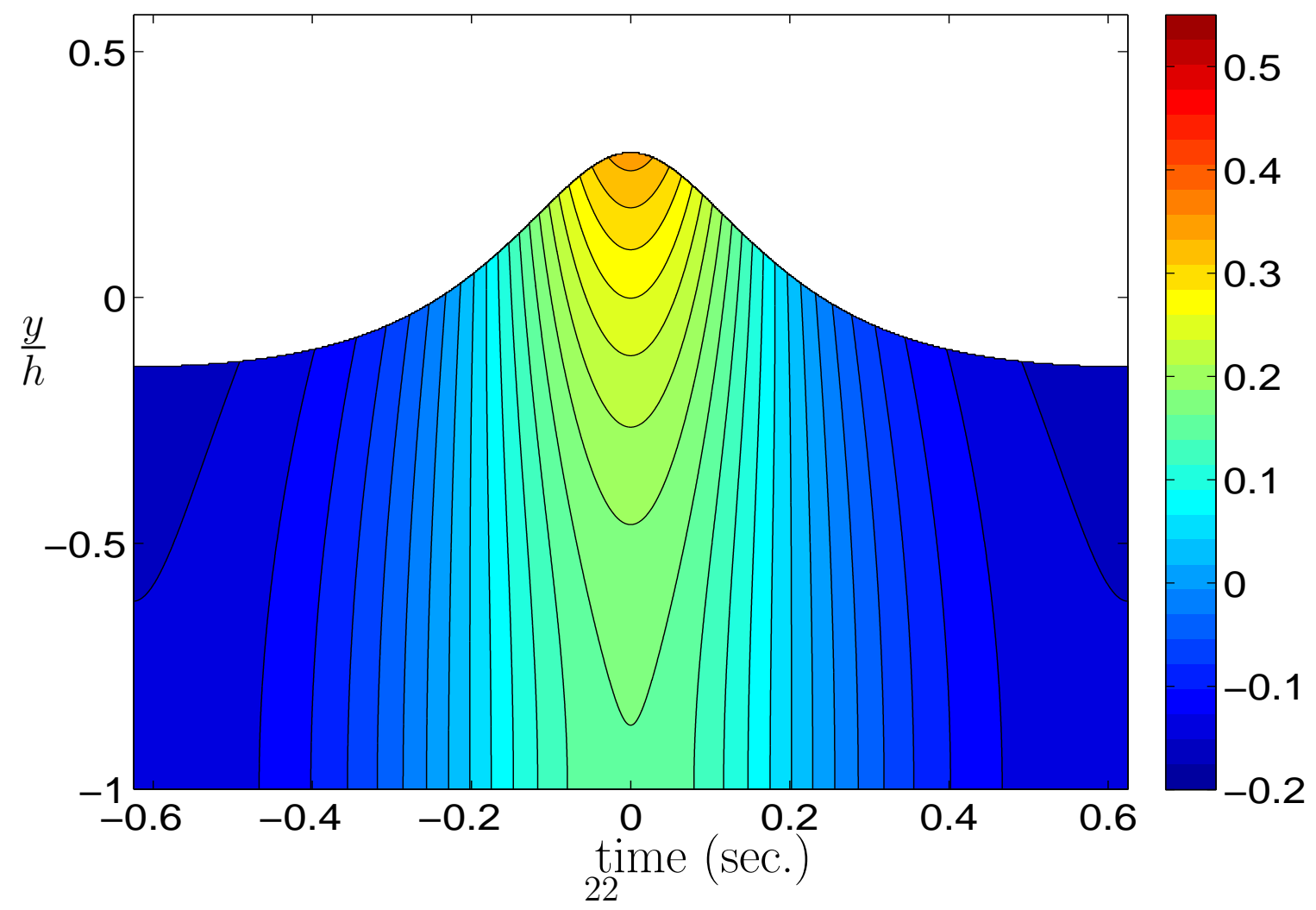


c)

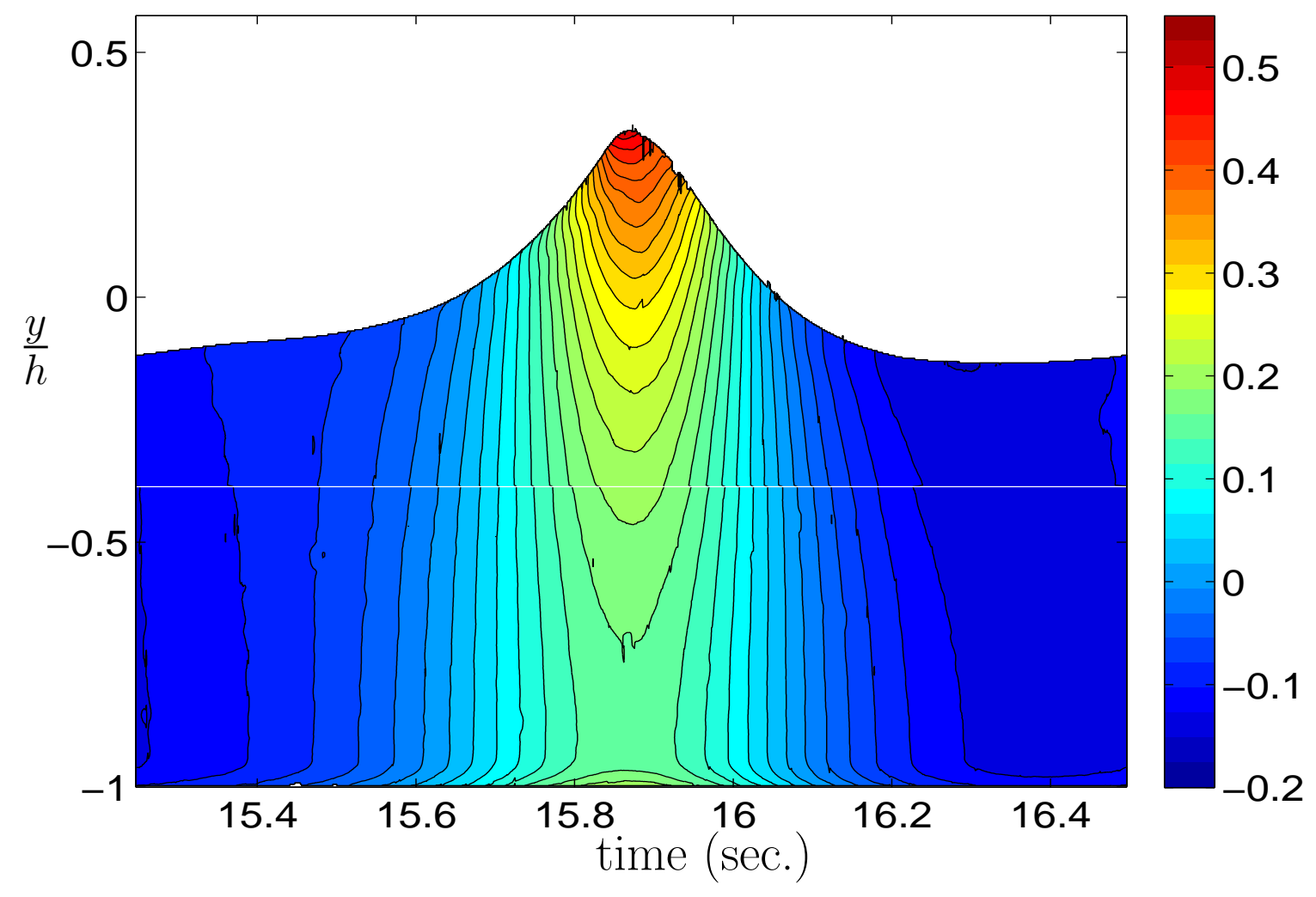

d)

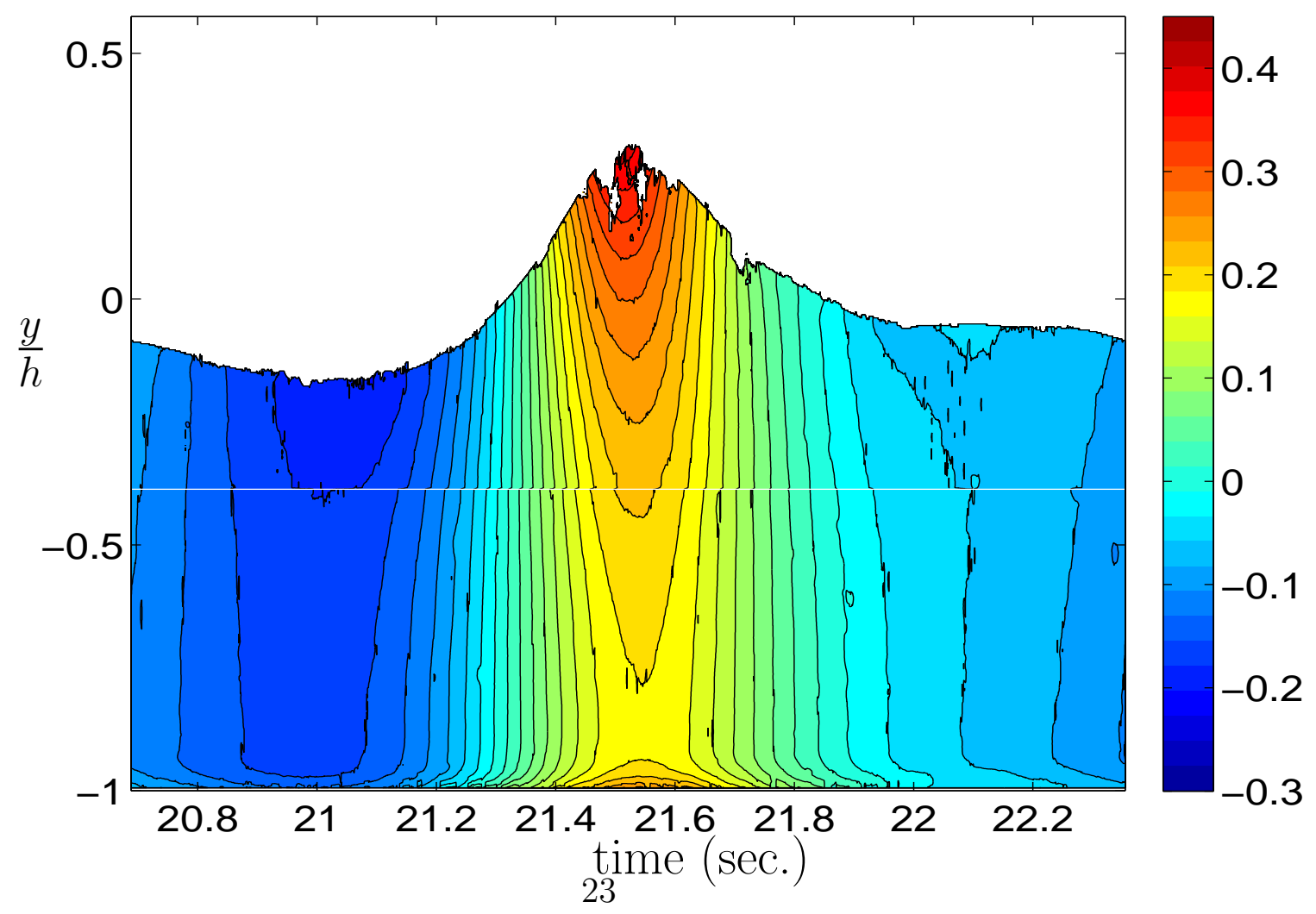

Figure 2: Elevation $\eta / h$ and horizontal velocity $u / \sqrt{g h}$ (isolines/color scale) by PIV. a) Same as Figure 1a, for $6.5 \mathrm{~s}<t<7.75 \mathrm{~s}, \mathrm{~b}$ ) Computation (Fenton's method) of wave in a), c) Same as Figure 1a, for $15.25 \mathrm{~s}<t<16.5 \mathrm{~s}$, d) Same as Figure 1b, for $20.9 \mathrm{~s}<t<$ $22.3 \mathrm{~s}$. 
a)
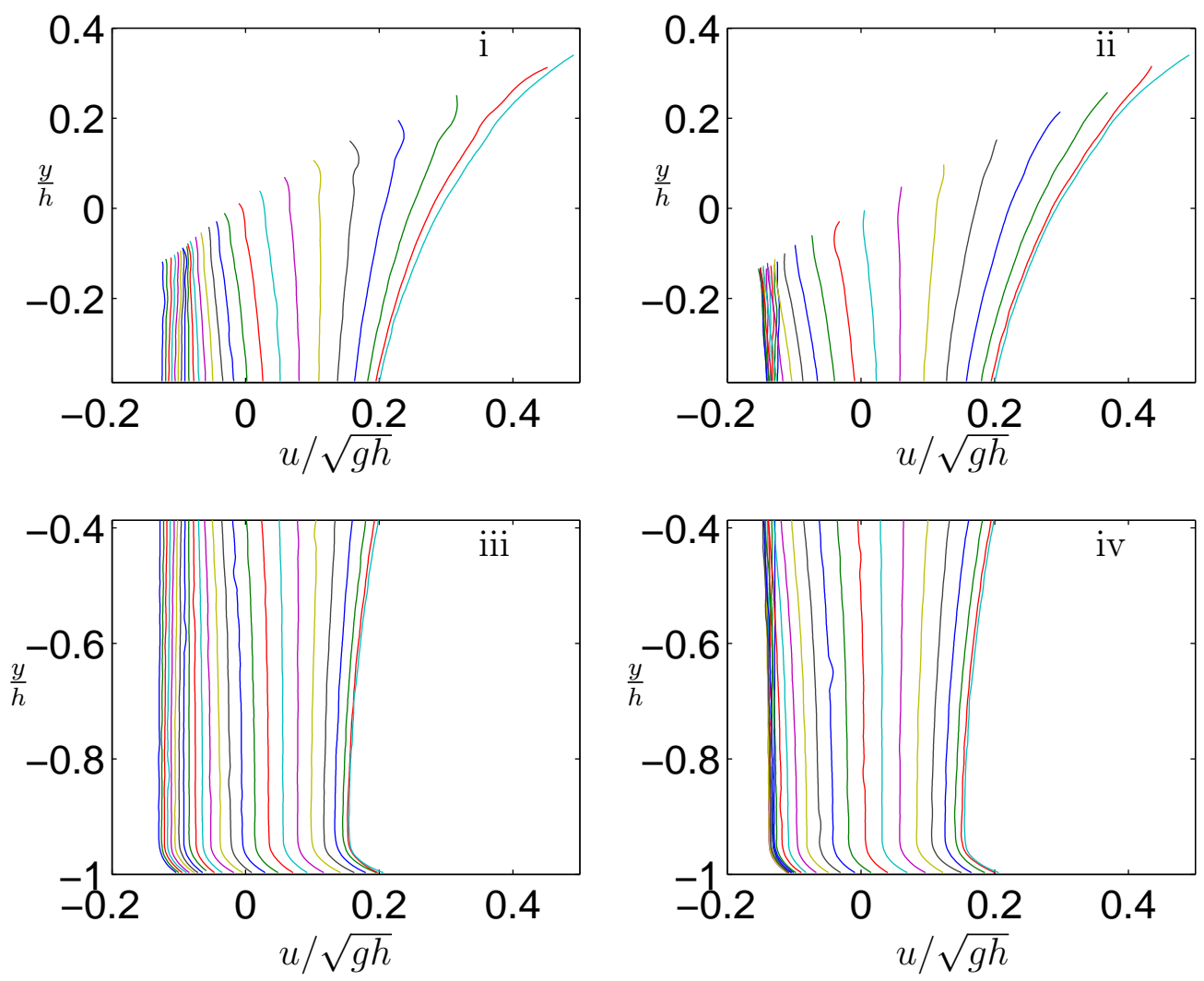

b)

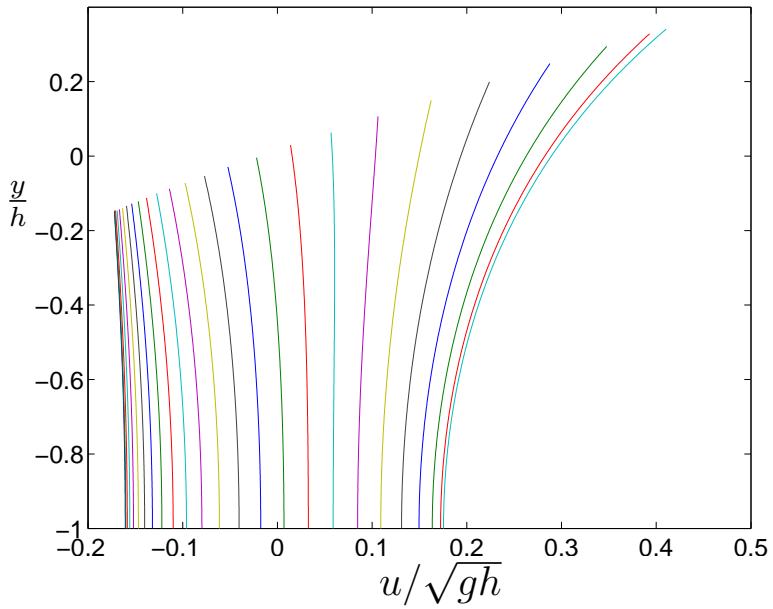


c)
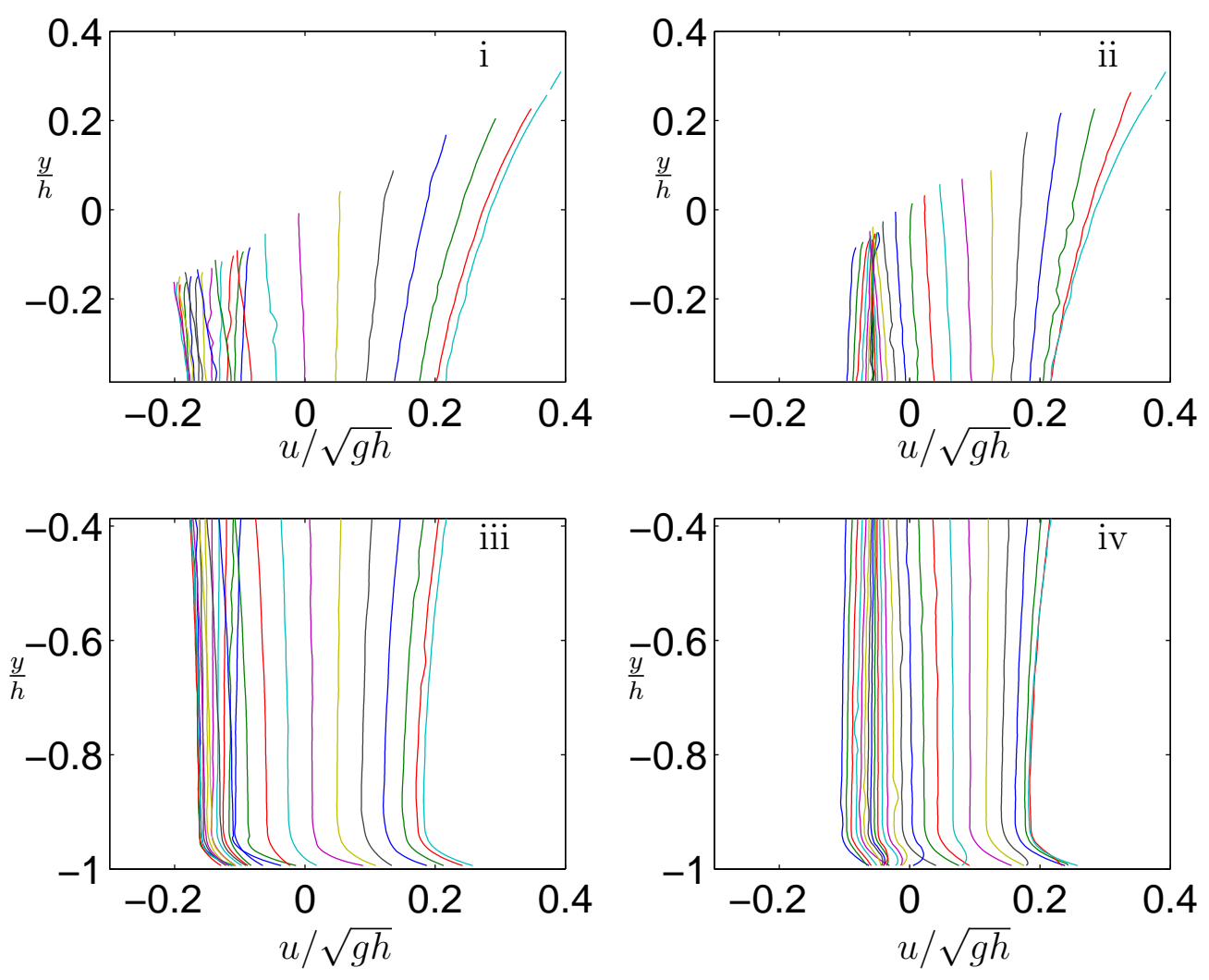

d)

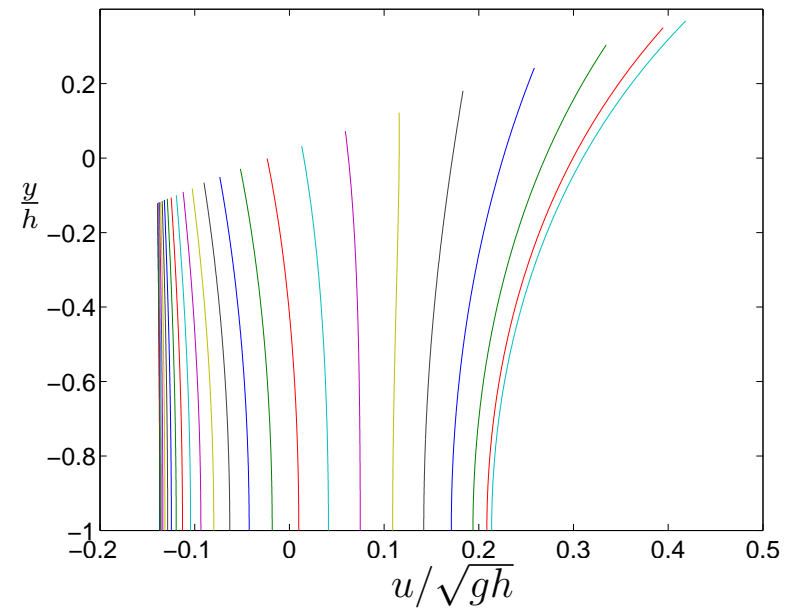


e)
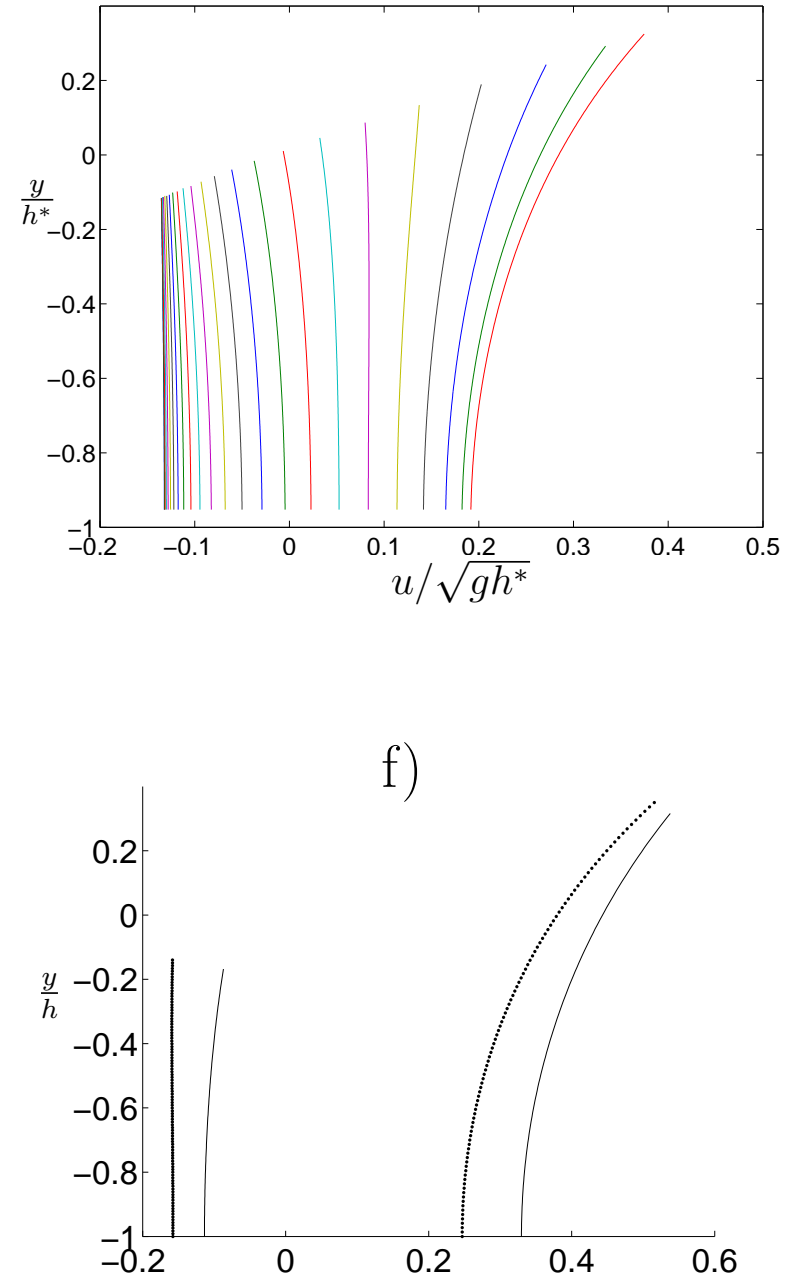

$u / \sqrt{g h}$

Figure 3: Velocity profiles $u / \sqrt{g h}$ vs. $y / h$. a) Experimental wave in Figure 2c), $\Delta t \sqrt{g / h}=$ 0.1821 (upper front (i), upper back (ii), lower front (iii), lower back (iv)). b) Computation wave in a). c) Experimental wave in Figure 2d), $\Delta t \sqrt{g / h}=0.2430$ (upper front (i), upper back (ii), lower front (iii), lower back (iv)). d) Computation of wave in c), with $H / h=0.48$, $T \sqrt{g / h}=11.7$. e) Computation of wave in c), with $H / h^{*}=0.466, T \sqrt{g / h^{*}}=11.4$. f) Velocity profiles at crest and trough by second order theory, $2 A / h=0.49 . T \sqrt{g / h}=8.75$ (thick dots), $T \sqrt{g / h}=11.7$ (line). 
a)

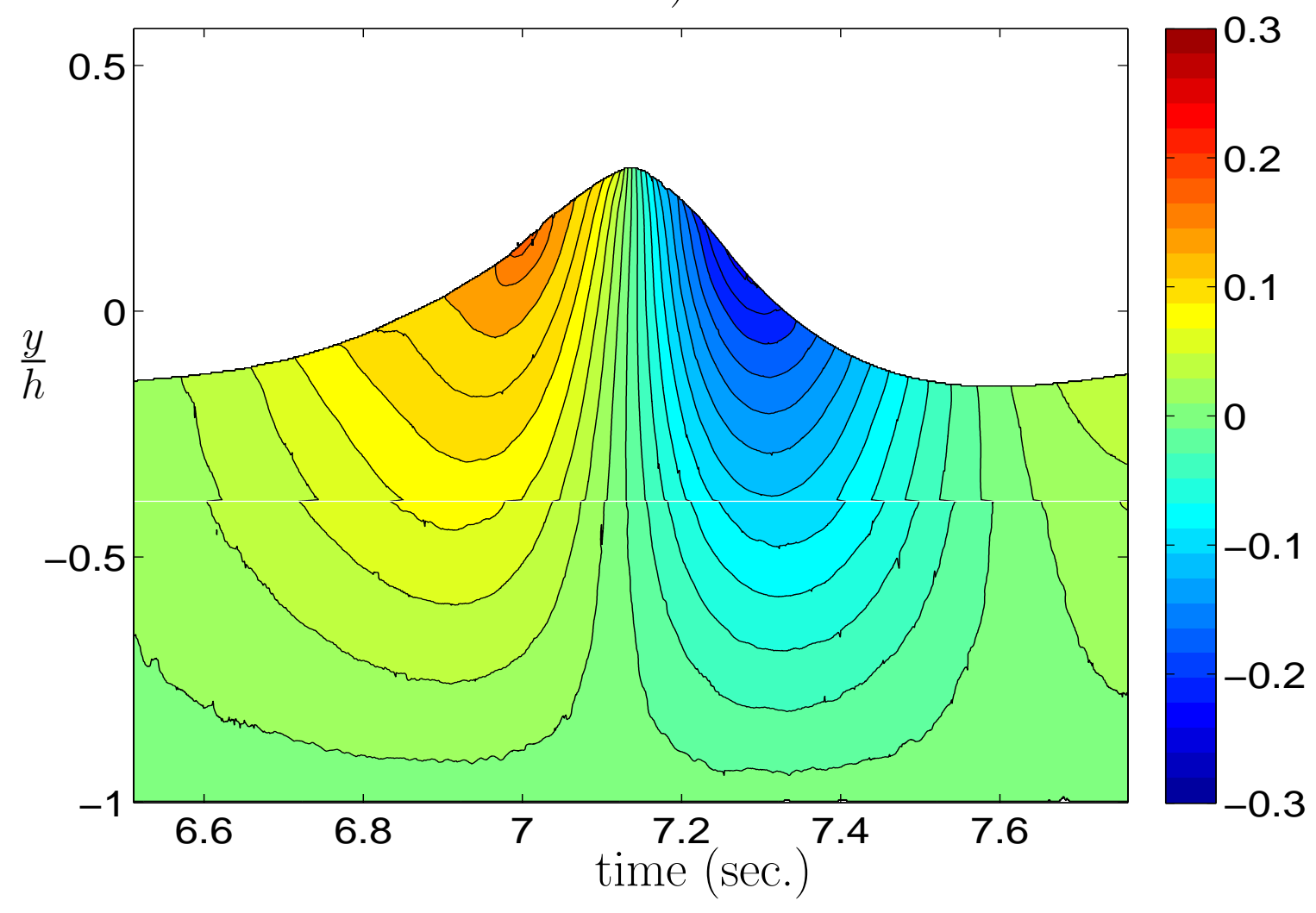

b)

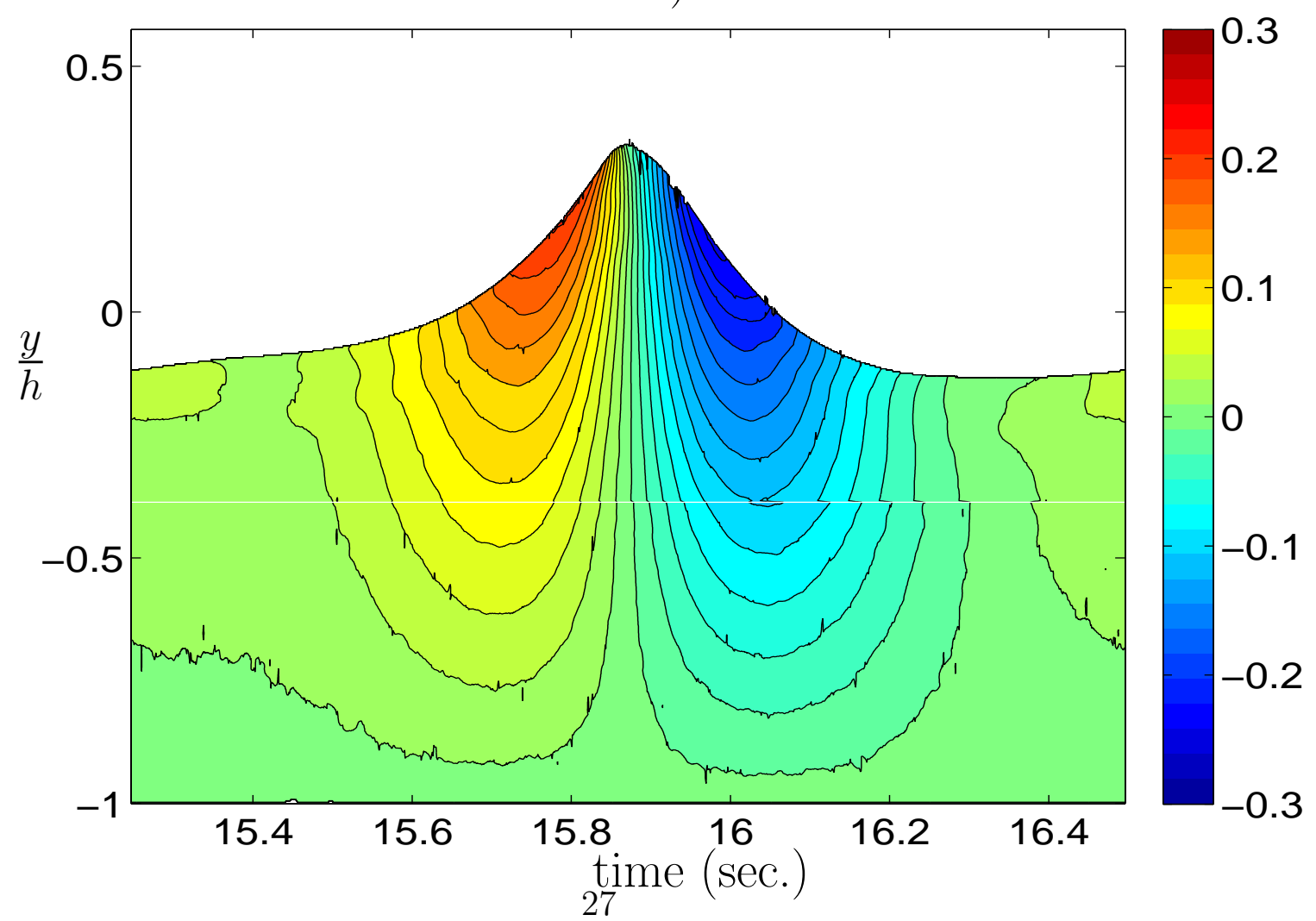

Figure 4: Vertical velocity $v / \sqrt{g h}$ (isolines/color scale) by PIV at $4.43 \mathrm{~m}$. $T \sqrt{g / h}=8.75$.

a) $6.5 \mathrm{~s}<t<7.75 \mathrm{~s}$, b) $15.25 \mathrm{~s}<t<16.5 \mathrm{~s}$. 


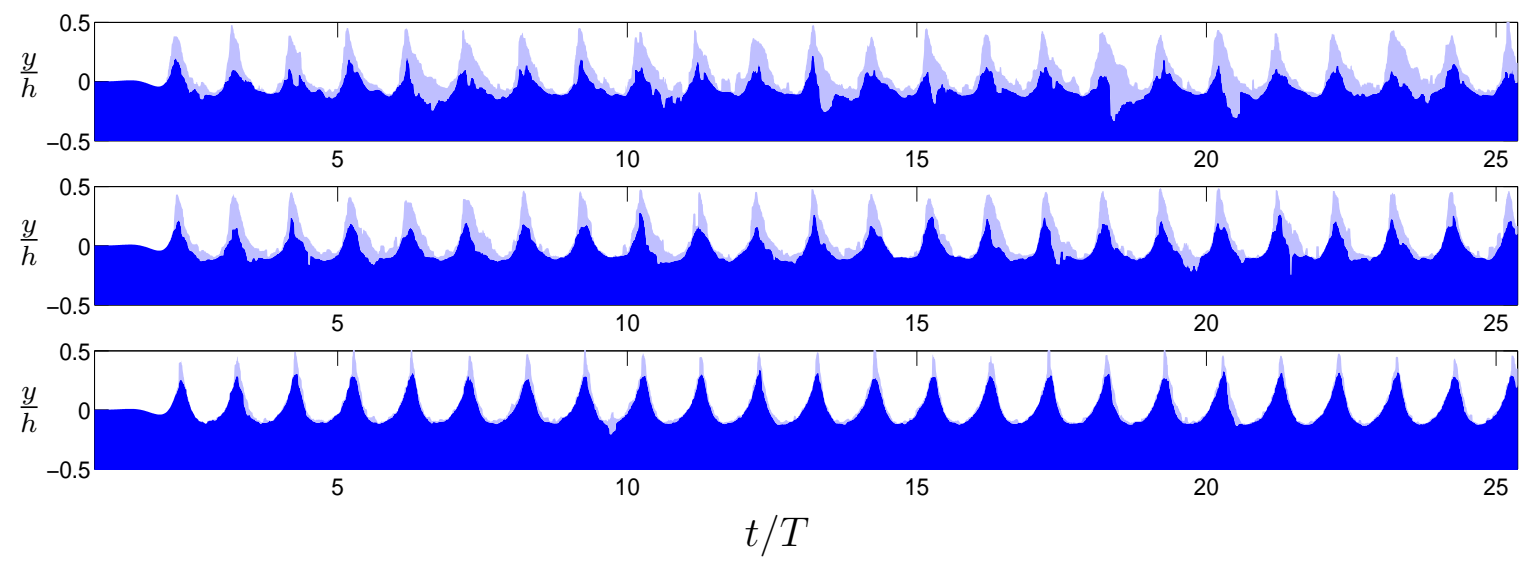

Figure 5: PIV recordings of breaking waves at $2.08 \mathrm{~m} . T \sqrt{g / h}=8.75 .24$ crests in each series. Smooth flow (dark). Breaking turbulent flow (light). Wave maker amplitude: a) $\xi_{0} / h=0.3697$, b) $\xi_{0} / h=0.3287$, c) $\xi_{0} / h=0.2876$. 
a)

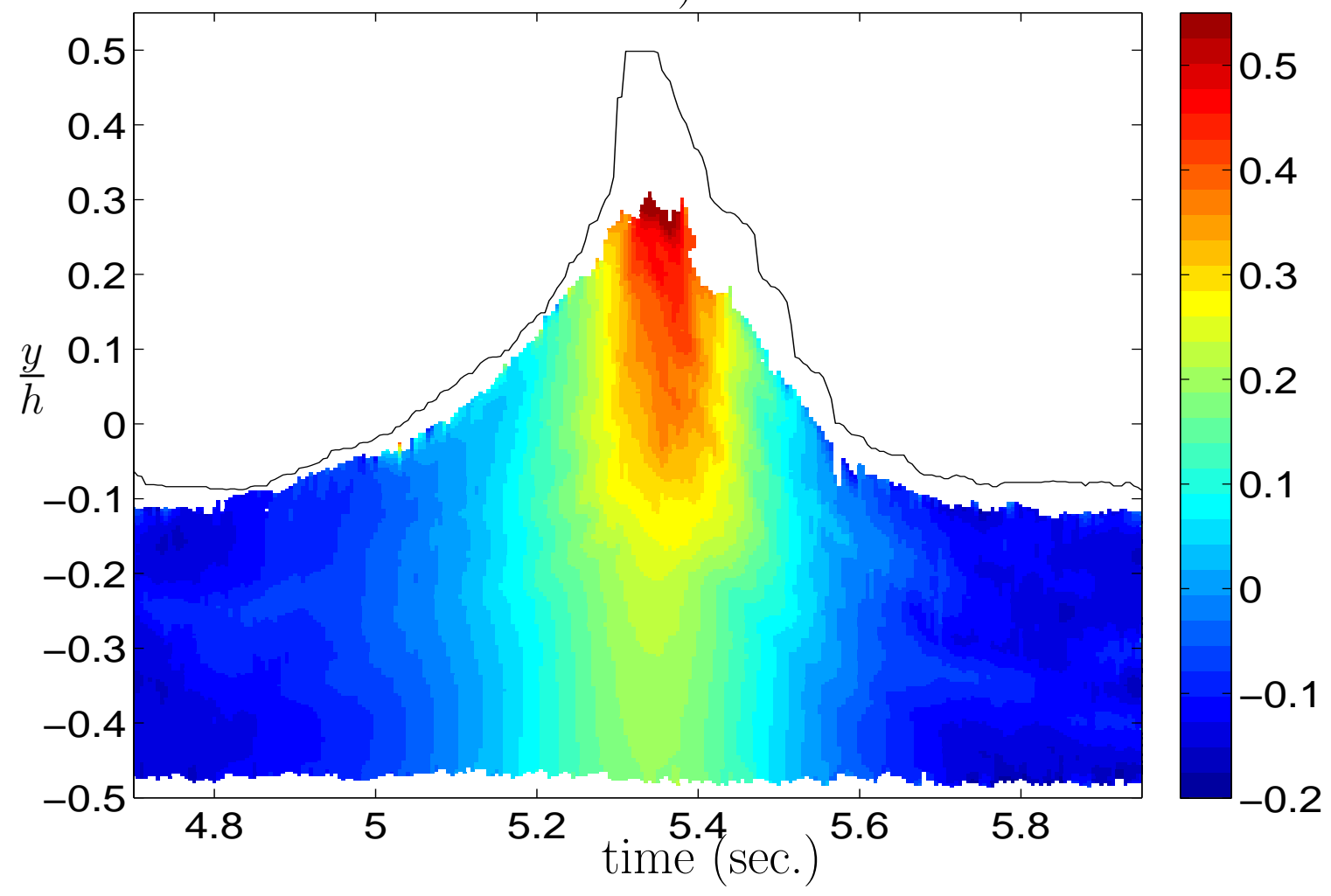

b)

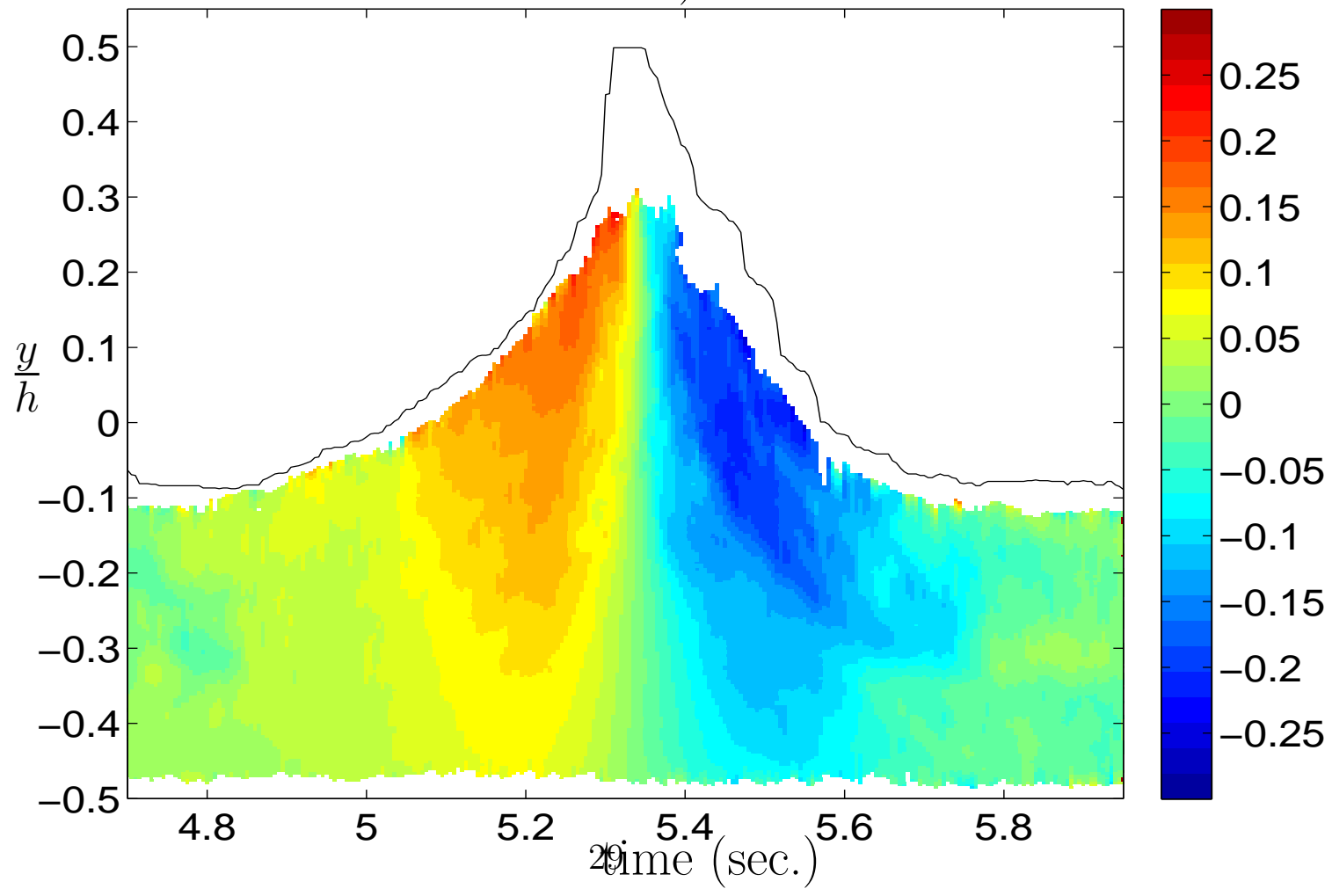


c)

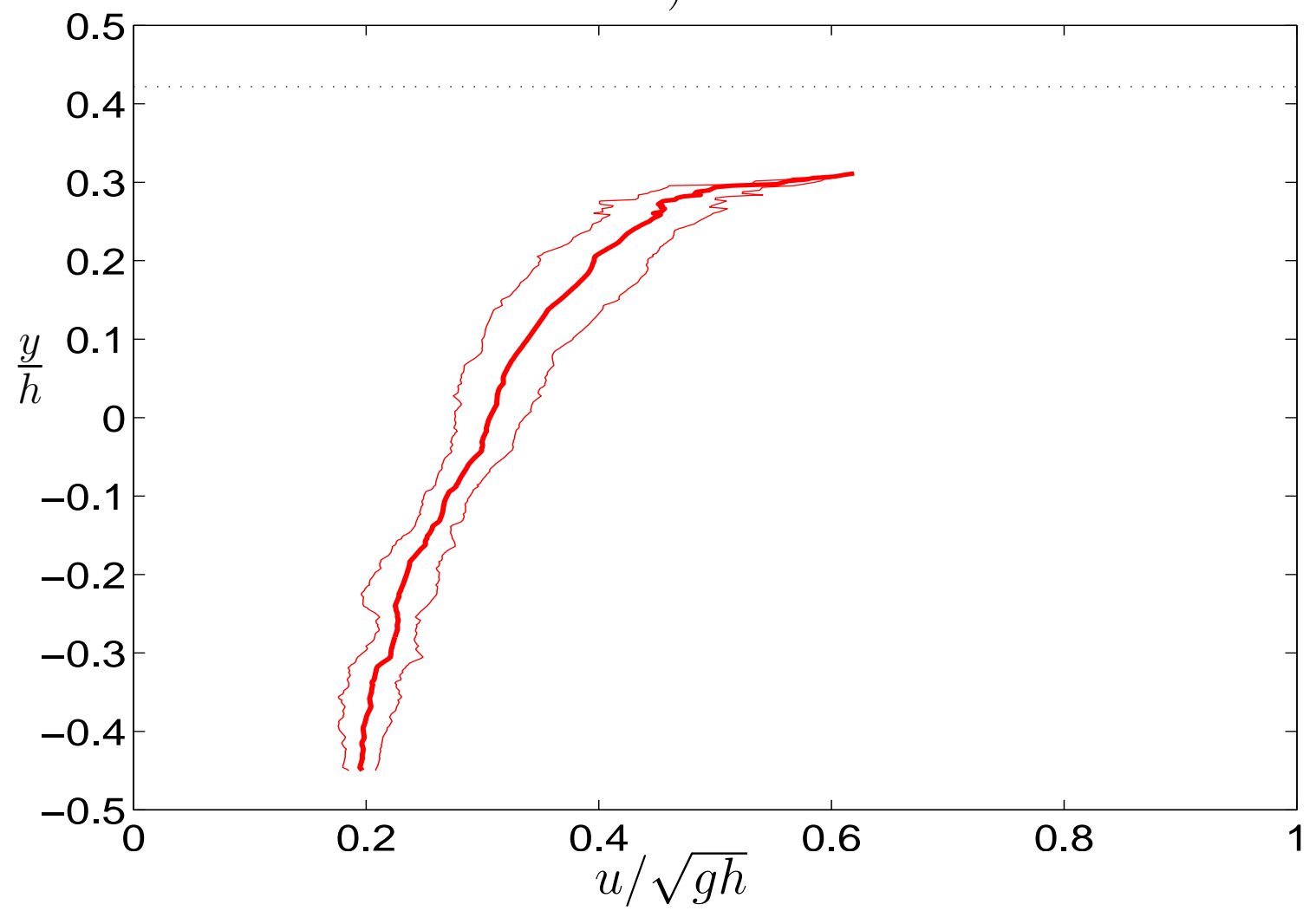

Figure 6: Elevation and $u / \sqrt{g h}$ (a) and $v / \sqrt{g h}$ (b) (color scale) for crest number three in figure $5 \mathrm{a}(4.7 \mathrm{~s}<t<5.95 \mathrm{~s})$. Moderately breaking wave. c) Ensemble average (thick line) and standard deviation (thin lines) of all velocity profiles $u / \sqrt{g h}$ vs. $y / h$ below the 24 crests in figure $5 \mathrm{c}$. 
a)
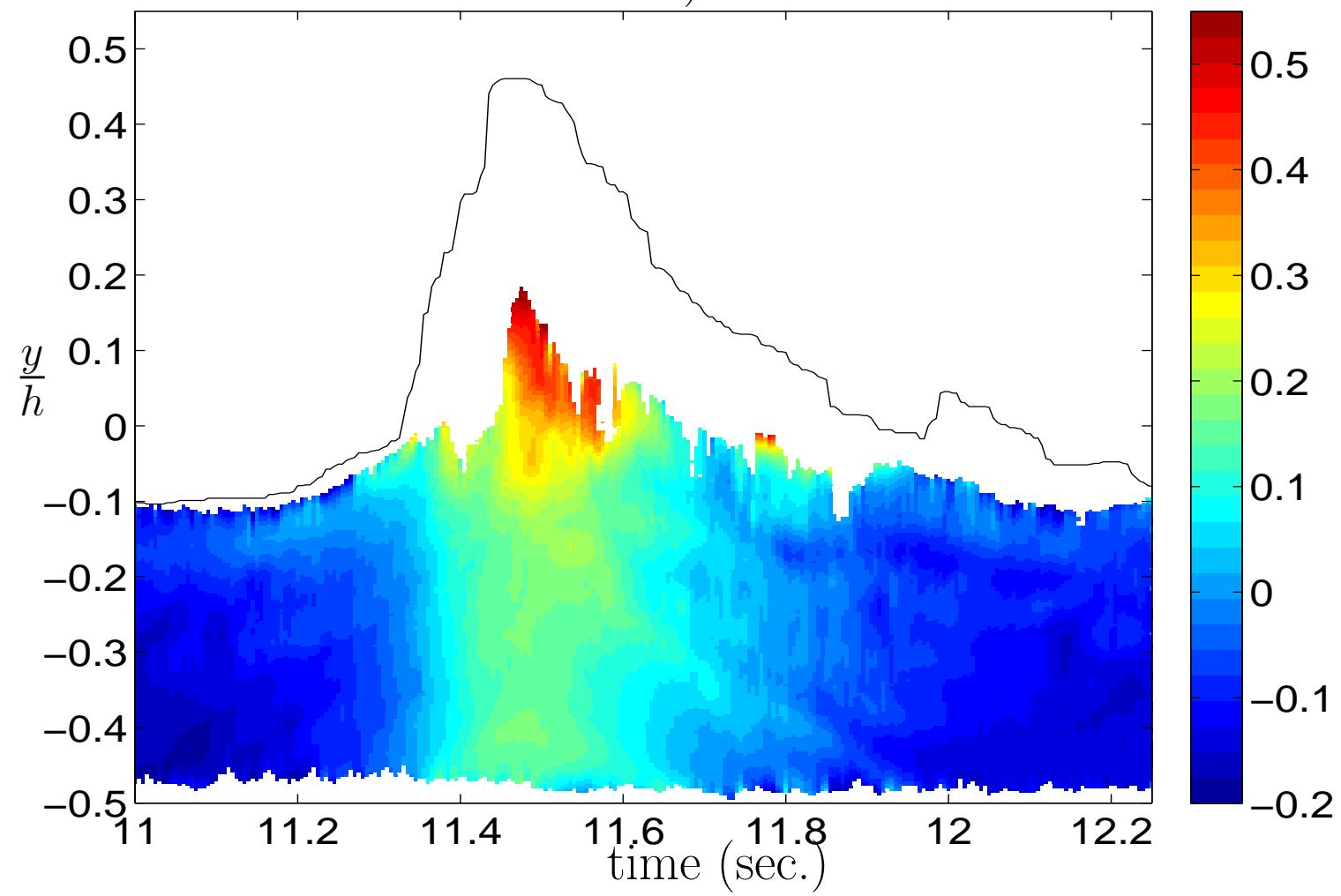

b)

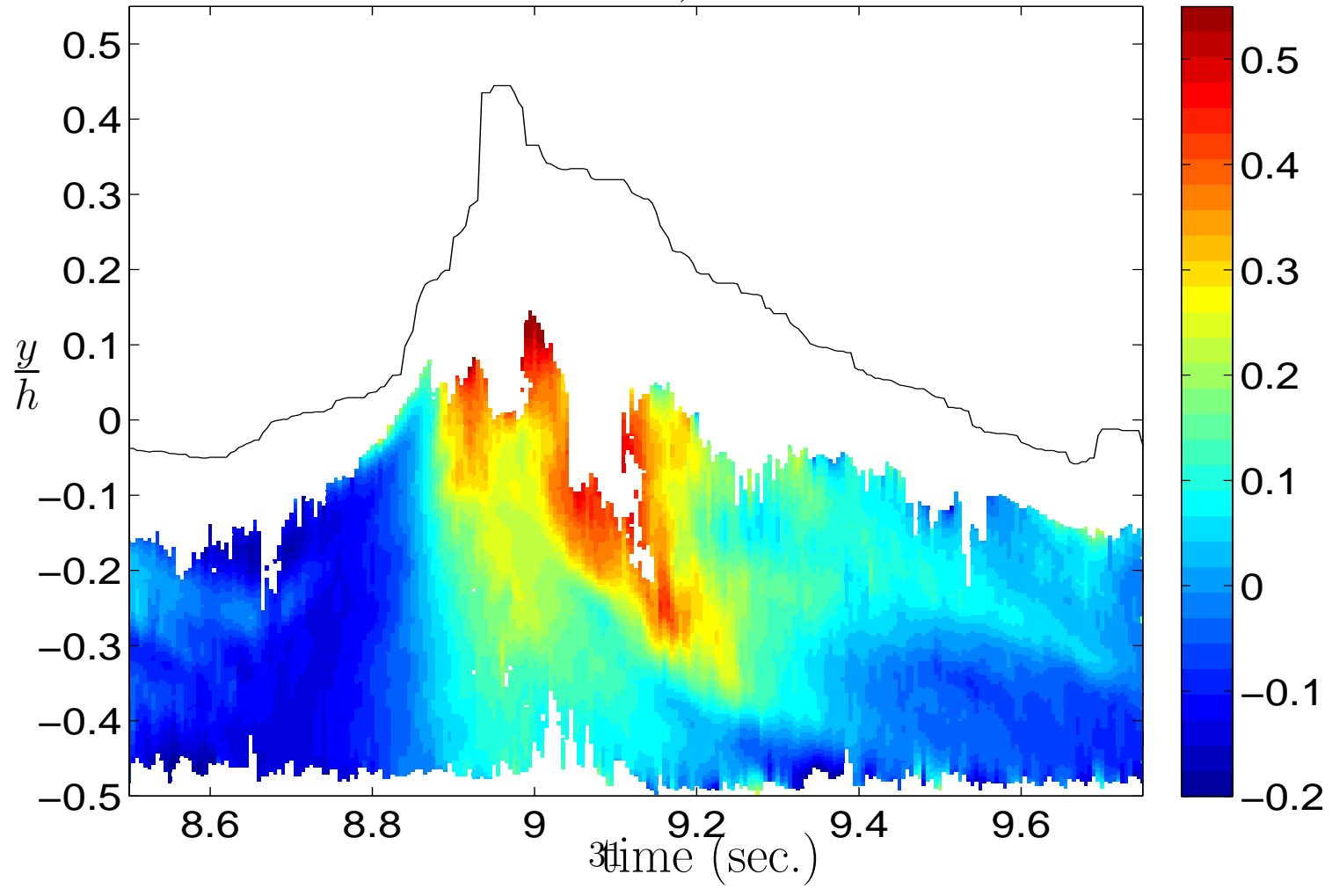



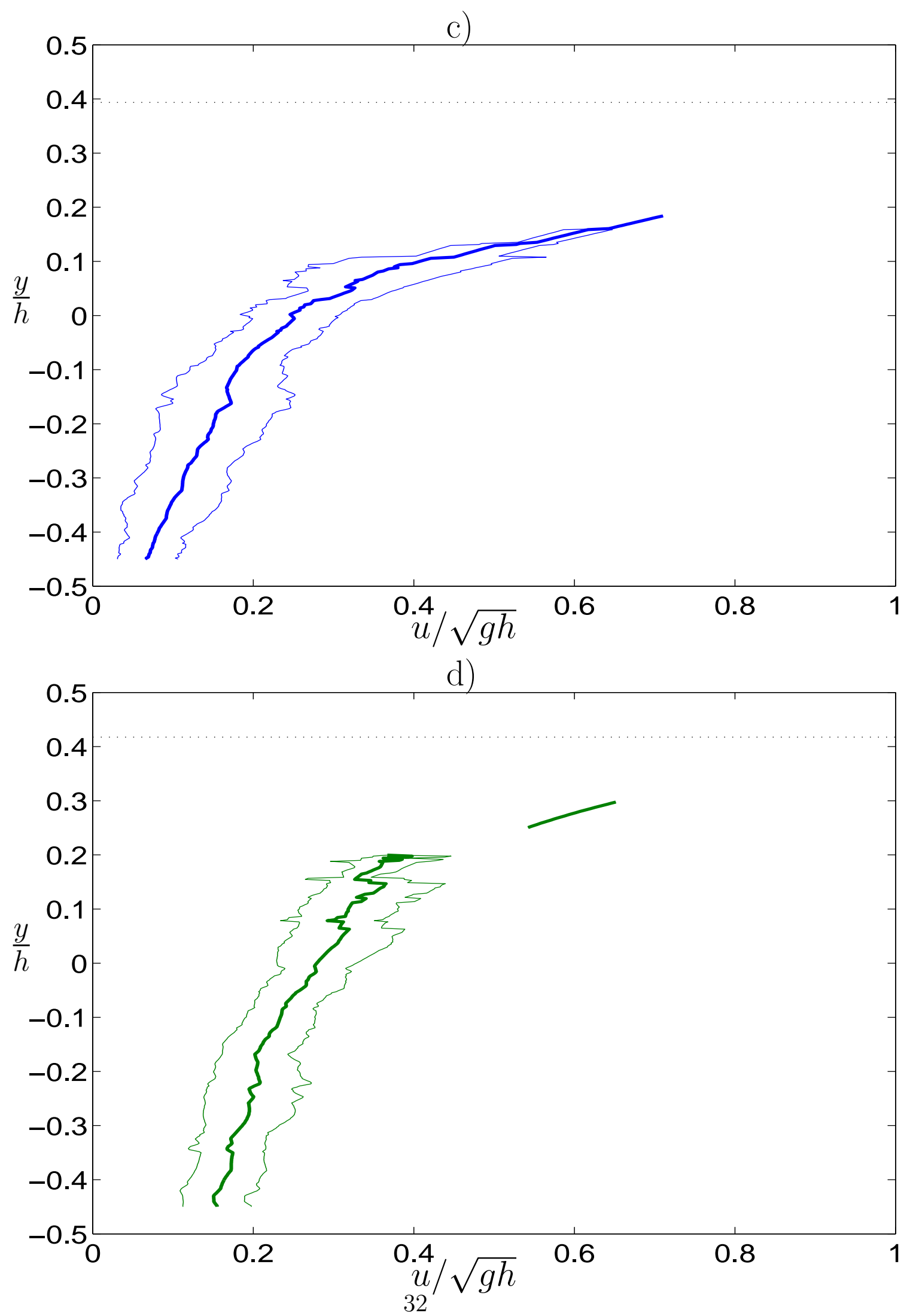

Figure 7: Same as figure 6a but for strongly breaking wave series. a) crest 8 figure 5 a (11 $\mathrm{s}<t<12.25 \mathrm{~s}), \mathrm{b})$ crest 6 in figure $5 \mathrm{a}(8.6 \mathrm{~s}<t<9.75 \mathrm{~s})$. c) same as figure $6 \mathrm{c}$ but for series in Figure 5a. d) same as figure $6 \mathrm{c}$ but for series in Figure $5 \mathrm{~b}$. 
a)
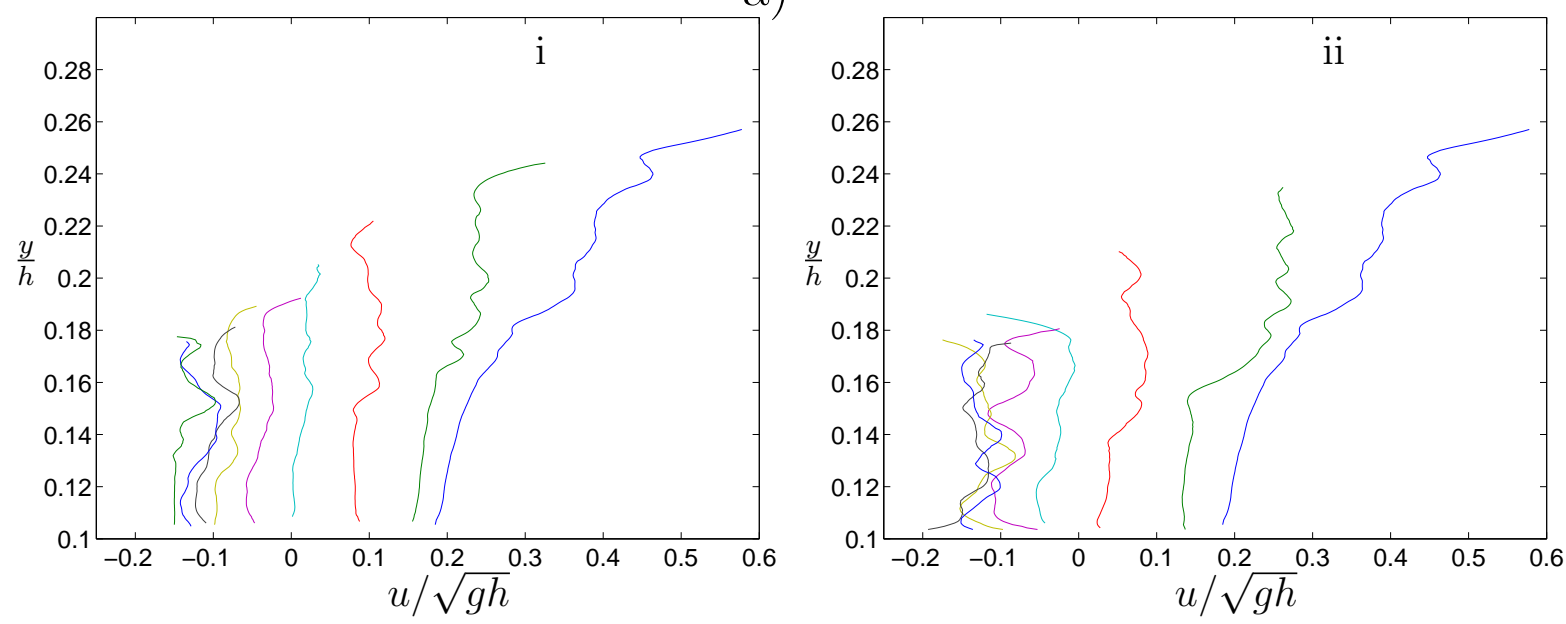

b)
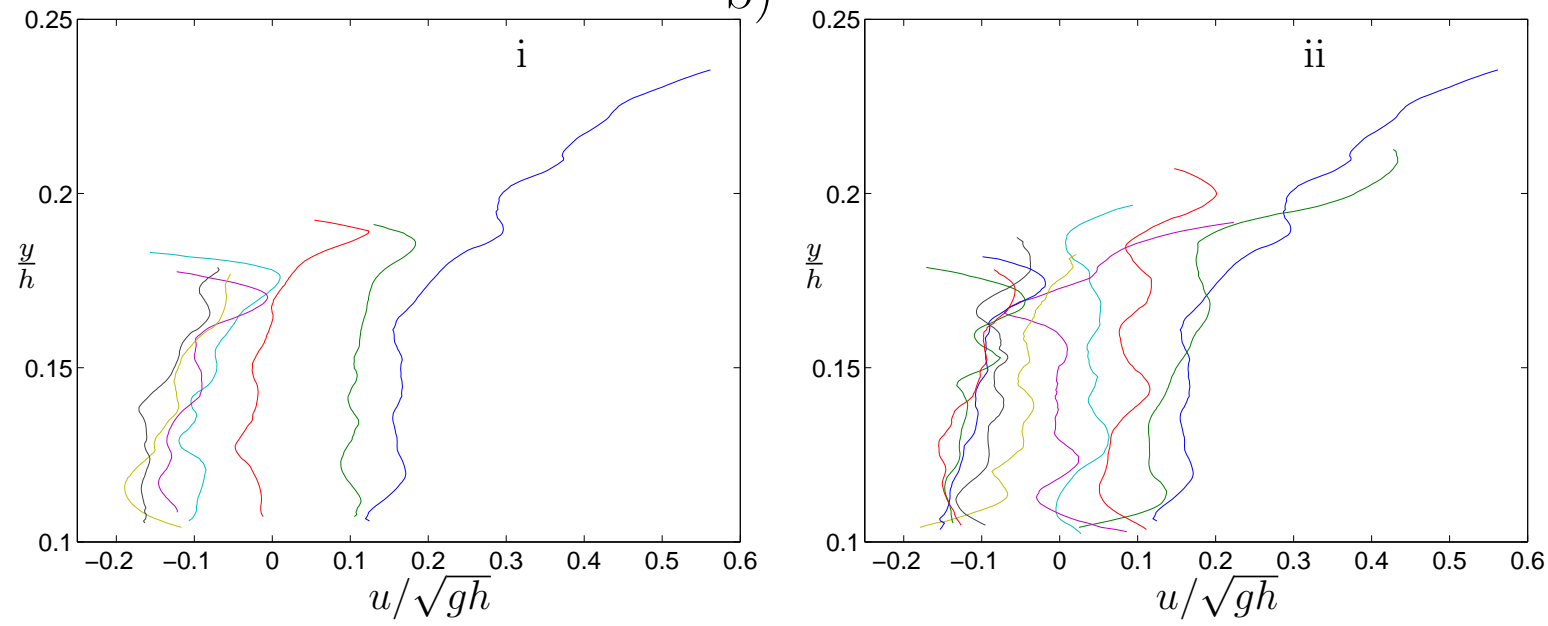

c)
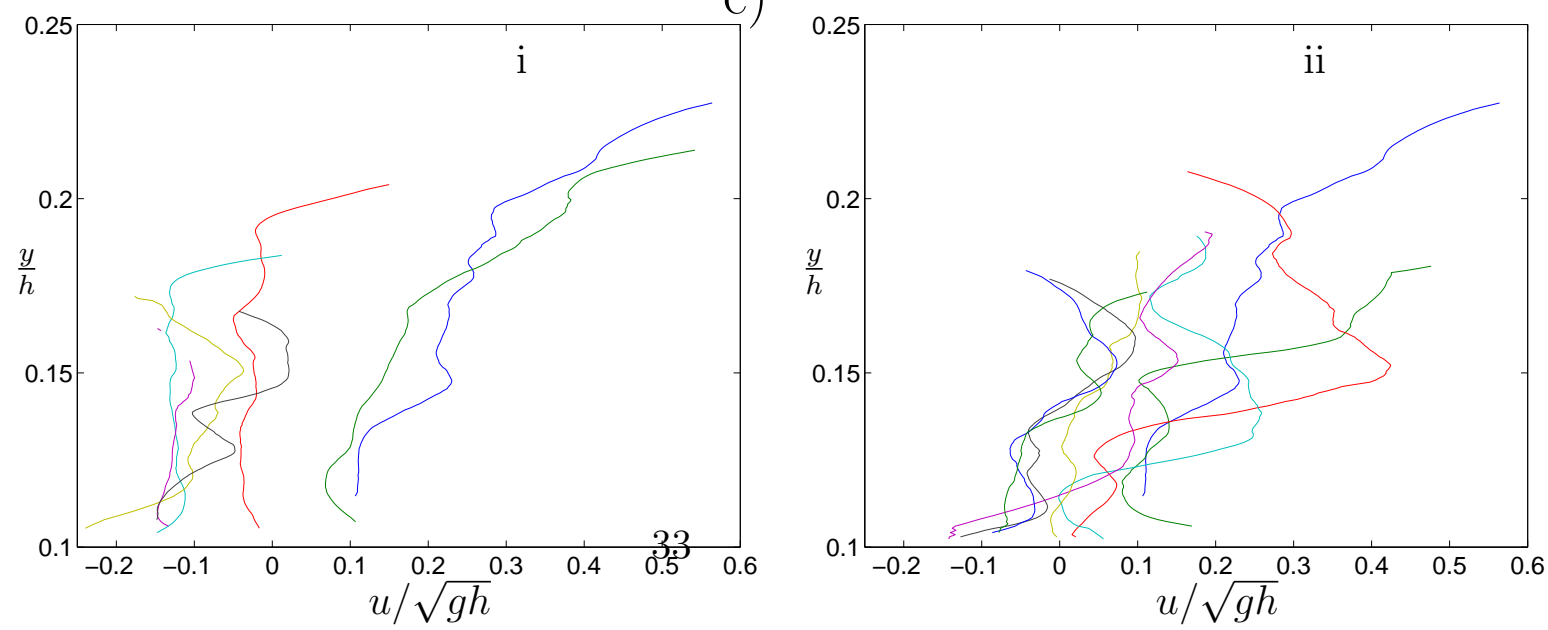

Figure 8: Horizontal velocity profiles at time intervals $\Delta t \sqrt{g / h}=1.75$. (i) front, (ii) back.

Wave in a) Figure 6a. b) Figure 7a. c) Figure 7b. 


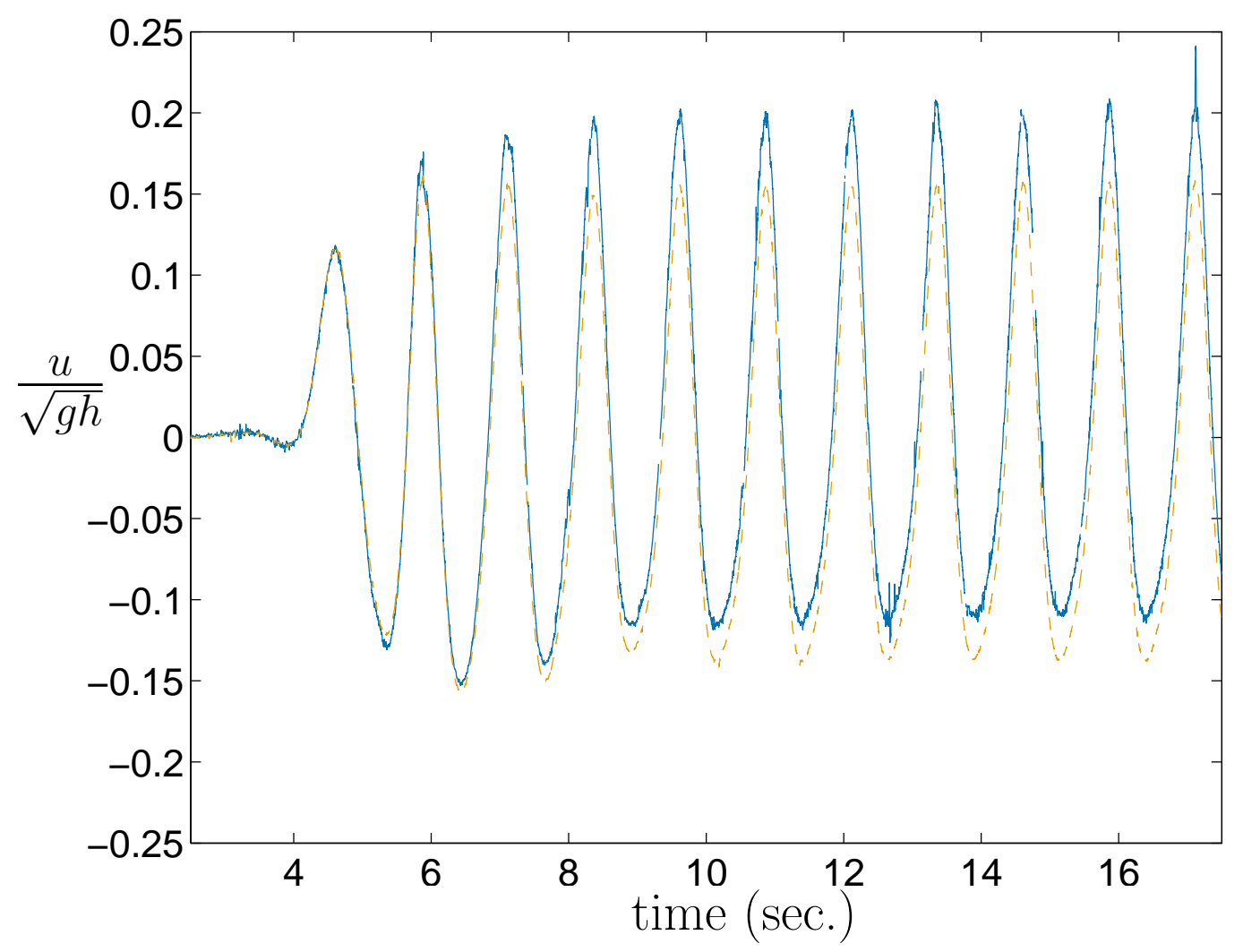

Figure 9: $u / \sqrt{g h}$ at $y / h=-0.96(--)$ and $y / h=-0.996(-)$ vs. time at the bottom, below the waves in figure $1 \mathrm{a}$. 